\title{
THE NEVAI CONDITION
}

\author{
JONATHAN BREUER ${ }^{1}$, YORAM LAST ${ }^{2,4}$, AND BARRY SIMON ${ }^{3,4}$
}

\begin{abstract}
We study Nevai's condition that for orthogonal polynomials on the real line, $K_{n}\left(x, x_{0}\right)^{2} K_{n}\left(x_{0}, x_{0}\right)^{-1} d \rho(x) \rightarrow \delta_{x_{0}}$ where $K_{n}$ is the CD kernel. We prove that it holds for the Nevai class of a finite gap set uniformly on the spectrum and we provide an example of a regular measure on $[-2,2]$ where it fails on an interval.
\end{abstract}

\section{INTRODUCTION}

This paper studies material on the borderline of the theory of orthogonal polynomials on the real line (OPRL) and spectral theory. Let $d \rho$ be a measure on $\mathbb{R}$ of compact but not finite support and let $P_{n}(x, d \rho), p_{n}(x, d \rho)$ be the standard [45, 15, 43] monic and normalized orthogonal polynomials for $d \rho$. Let $\left\{a_{n}, b_{n}\right\}_{n=1}^{\infty}$ be the Jacobi parameters defined by

$$
x p_{n}(x)=a_{n+1} p_{n+1}(x)+b_{n+1} p_{n}(x)+a_{n} p_{n-1}(x)
$$

The CD (for Christoffel-Darboux) kernel is defined by

$$
K_{n}(x, y)=\sum_{j=0}^{n} p_{j}(x) p_{j}(y)
$$

for $x, y$ real. The CD formula (see, e.g., [41]) asserts that

$$
K_{n}(x, y)=\frac{a_{n+1}\left[p_{n+1}(x) p_{n}(y)-p_{n}(x) p_{n+1}(y)\right]}{x-y}
$$

Date: September 4, 2008.

2000 Mathematics Subject Classification. 42C05, 39A10, 30C10.

Key words and phrases. Orthogonal polynomials, regular measures, CD kernel.

${ }^{1}$ Mathematics 253-37, California Institute of Technology, Pasadena, CA 91125, USA. E-mail: jbreuer@caltech.edu.

${ }^{2}$ Institute of Mathematics, The Hebrew University, 91904 Jerusalem, Israel. Email: ylast@math.huji.ac.il. Supported in part by The Israel Science Foundation (grant no. 1169/06).

${ }^{3}$ Mathematics 253-37, California Institute of Technology, Pasadena, CA 91125, USA. E-mail: bsimon@caltech.edu. Supported in part by NSF grant DMS-0652919.

${ }^{4}$ Research supported in part by Grant No. 2006483 from the United States-Israel Binational Science Foundation (BSF), Jerusalem, Israel. 
The Christoffel variational principle (see [41]) says that if

$$
\lambda_{n}\left(x_{0}\right)=\min \left\{\int\left|Q_{n}(x)\right|^{2} d \rho(x) \mid \operatorname{deg} Q_{n} \leq n, Q_{n}\left(x_{0}\right)=1\right\}
$$

then

$$
\lambda_{n}\left(x_{0}\right)=\frac{1}{K_{n}\left(x_{0}, x_{0}\right)}
$$

and the minimizer is given by

$$
\widetilde{Q}_{n}\left(x, x_{0}\right)=\frac{K_{n}\left(x, x_{0}\right)}{K_{n}\left(x_{0}, x_{0}\right)}
$$

It is quite natural to look at the probability measures

$$
d \eta_{n}^{\left(x_{0}\right)}(x)=\frac{\left|\widetilde{Q}_{n}\left(x, x_{0}\right)\right|^{2} d \rho(x)}{\int\left|\widetilde{Q}_{n}\left(y, x_{0}\right)\right|^{2} d \rho(y)}
$$

so that, by (1.5) and (1.6),

$$
d \eta_{n}^{\left(x_{0}\right)}=\frac{\left|K_{n}\left(x, x_{0}\right)\right|^{2} d \rho(x)}{K_{n}\left(x_{0}, x_{0}\right)}
$$

Definition. We say $d \rho$ obeys a Nevai condition at $x_{0}$ if and only if

$$
\underset{n \rightarrow \infty}{\mathrm{w}-\lim _{n}} d \eta_{n}^{\left(x_{0}\right)}=\delta_{x_{0}}
$$

the point mass at $x_{0}$.

The name comes from the fact that this condition was studied in the seminal work of Nevai [32], who considered the following:

Definition. The Nevai class for $[-2,2]$ is the set of all measures $d \rho$ whose Jacobi parameters obey

$$
a_{n} \rightarrow 1 \quad b_{n} \rightarrow 0
$$

$[-2,2]$ is relevant since, by Weyl's theorem on essential spectrum, $[-2,2]$ is the derived set of $\operatorname{supp}(d \rho)$, that is, the essential spectrum for the Jacobi matrix of $d \rho$.

Nevai proved the following:

Theorem 1.1 (Nevai [32]). If $d \rho$ is in the Nevai class for $[-2,2]$, then the Nevai condition holds for all $x_{0}$ in $[-2,2]$. Indeed, the limit is uniform for $x_{0}$ in any compact set $K \subset(-2,2)$.

The connection of this to spectral theory comes from the relation to the following condition, sometimes called subexponential growth,

$$
\lim _{n \rightarrow \infty} \frac{\left|p_{n}\left(x_{0}\right)\right|^{2}}{\sum_{j=0}^{n}\left|p_{j}\left(x_{0}\right)\right|^{2}}=0
$$


which we will show (see Proposition 2.1) is equivalent to

$$
\lim _{n \rightarrow \infty} \frac{\left(\left|p_{n-1}\left(x_{0}\right)\right|^{2}+\left|p_{n}\left(x_{0}\right)\right|^{2}\right)}{\sum_{j=0}^{n}\left|p_{j}\left(x_{0}\right)\right|^{2}}=0
$$

and to

$$
\lim _{n \rightarrow \infty} \frac{\left|p_{n+1}\left(x_{0}\right)\right|^{2}}{\sum_{j=0}^{n}\left|p_{j}\left(x_{0}\right)\right|^{2}}=0
$$

We will sometimes need

$$
0<A_{-} \equiv \inf _{n} a_{n} \leq \sup _{n} a_{n} \equiv A_{+}<\infty
$$

We note $A_{+}<\infty$ follows from the assumption that $\operatorname{supp}(d \rho)$ is compact, and if $A_{-}=0$, then by a result of Dombrowski [14], $d \mu$ is purely singular with respect to Lebesgue measure. Obviously, (1.14) holds for the discrete Schrödinger case, $a_{n} \equiv 1$.

The relation of (1.11) to Nevai's condition is direct:

Theorem 1.2. Let (1.14) hold. Nevai's condition holds at $x_{0}$ if and only if (1.11) holds.

That $(1.11) \Rightarrow(1.9)$ is due to Nevai. The converse is new here and appears as Theorem 2.2 .

Equation (1.11) is, of course, the kind of asymptotic eigenfunction result of interest to spectral theorists and is susceptible to the methods of spectral theory. In particular, Theorem 1.2 shows that for compactly supported measures, the Nevai condition is intimately connected with the existence of certain natural sequences of approximate eigenvectors for the associated Jacobi matrix (for the relevance of approximate eigenvectors to spectral analysis see, e.g., [5]). Note that (1.1) and the orthogonality relation say that, for $\rho$-a.e. $x$, the sequence $\left(p_{0}(x), p_{1}(x), \ldots\right)$ is a generalized eigenfunction, at $x$, for the Jacobi matrix, $J$, defined by the parameters $\left\{a_{n}, b_{n}\right\}_{n=1}^{\infty}$ (namely, it is a polynomially bounded solution of the corresponding eigenvalue equation). Truncations of generalized eigenfunctions are natural candidates for sequences of approximate eigenvectors, and Theorem 1.2 (see (2.13) in particular) says that the Nevai condition at $x_{0}$ is equivalent to the requirement that truncations of the generalized eigenfunction at $x_{0}$ yield a sequence of approximate eigenvectors for $J$. In fact, from the point of view of spectral theory, for compactly supported measures, the Nevai condition is a simple restatement of this in the "energy representation" of the Jacobi matrix.

This connection is one reason Nevai's condition is interesting - it has also been used to relate ratios of $\lambda\left(x_{0}\right)$ for the measures $d \rho(x)$ and 
$e^{g(x)} d \rho(x)$ (Nevai's motivation in [32]). In this context, it was used by Máté-Nevai-Totik [31] to relate CD kernel asymptotics for OPUC and OPRL and to study OP asymptotics when a Szegö condition fails (see [28, 29, 30]; see also [43, Sect. 3.10]).

Given the form of the Christoffel variational principle, Nevai's condition seems like something that must always hold for $x_{0} \in \operatorname{supp}(d \mu)$. However, (1.11) also provides a basis for some counterexamples to Nevai's condition. In Section 3 (see Theorem 3.2), we will prove that

Theorem 1.3. If

$$
\liminf _{n \rightarrow \infty}\left(\left|p_{n}\left(x_{0}\right)\right|^{2}+\left|p_{n+1}\left(x_{0}\right)\right|^{2}\right)^{1 / n}>1
$$

then (1.11) fails.

This was the basis for the first counterexample to (1.11) by Szwarc 46]; in Section 3. we will see that the Anderson model provides an example of a measure on $[-2,2]$ for which the Nevai condition fails for Lebesgue a.e. $x_{0} \in[-2,2]$ !

Of course, (1.15) is associated with positive Lyapunov exponent. One might guess that zero Lyapunov exponent implies (1.11). A main impetus for this paper was our realization that this is not true! Recall that a measure on $[-2,2]$ is called regular if and only if $\lim \left(a_{1} \cdots a_{n}\right)^{1 / n}=1$ (see Stahl-Totik [44] or Simon [39] for reviews) and that regular measures have zero Lyapunov exponent, that is, for quasi-every (namely, outside, possibly, a set of zero logarithmic capacity) $x_{0} \in[-2,2]$,

$$
\lim _{n \rightarrow \infty} \frac{1}{n} \log \left(\left|p_{n}\left(x_{0}\right)\right|^{2}+\left|p_{n+1}\left(x_{0}\right)\right|^{2}\right)^{1 / 2}=0
$$

In Sections 4 and 5, we provide two examples of regular measures on $[-2,2]$ for which Nevai's condition fails for Lebesgue a.e. $x_{0}$ in $\pm(1,2)$. The example in Section 4 will be somewhat simpler but will have no a.c. spectrum, while that in Section 5 will have pure a.c. spectrum on $(-1,1)$.

We also want to discuss extensions of Nevai's theorem, Theorem 1.1. In this regard, we should mention some beautiful work of Nevai-TotikZhang [34] and Zhang [50] that already expanded this. The first paper proved uniform convergence on $[-2,2]$ with an elegant approach; this was extended in the second paper to $\left(a_{n}, b_{n}\right)$ approaching a periodic limit. Somewhat earlier, Lubinsky-Nevai [26] had proven the Nevai condition for this periodic limit case but only uniformly on compact subsets of the interior of the spectrum. That paper also has results on subexponential growth for some cases of measures that do not have 
compact support, a subject beyond the scope of this paper. Still later, another proof for the periodic limit case was found by Szwarc [47].

In the past four years, it has become clear that the proper analog of the Nevai class for the periodic case is not approach to a fixed periodic element but approach to an isospectral torus. We want to prove that not only can one do this in the periodic case, but on approach to the isospectral torus of, in general, almost periodic Jacobi matrices that occurs in the general finite gap case. We will also study the Nevai condition on the a.c. spectrum of ergodic Jacobi matrices and for the Fibonacci model.

From what we have said so far, it appears to be a mixed verdict on the Nevai condition since we have results on when it occurs and also on when it doesn't. But we want to reinterpret the negative results. These all provide examples where it is not true that the Nevai condition holds everywhere on the topological support of $d \rho$ or even that it fails for Lebesgue a.e. $x_{0}$ on the support. We believe it is likely that the following more refined property could be true:

Conjecture 1.4. For any measure, $d \rho$, with compact support, the Nevai condition holds for $d \rho$-a.e. $x_{0}$ in $\operatorname{supp}(d \rho)$.

Here is a summary of the contents of the rest of the paper. In Section 2, we prove Theorem 1.2. In Section 3, we discuss cases with positive Lyapunov exponent, including a proof of Theorem 1.3. Sections 4 and 5 present the details of the examples of regular measures where the Nevai condition fails for Lebesgue a.e. $x_{0}$ in a particular open subset. Section 6 presents our version of the NTZ approach, as preparation for our discussion of the finite gap Nevai class in Section 7 . In Section 8, we will relate (1.11) to the absence of $\ell^{2}$ solutions of classical right limits and so recover the results of Section 7 and even more (including Fibonacci models). The ideas of Section 8 seem to be more generally applicable than Section 6, but the constants in Section 6 are more explicit. Section 9 has some final remarks, including a discussion of Conjecture 1.4 and a second conjecture (Conjecture 9.5). Section 9 also notes that if $\frac{1}{n} K_{n}\left(x_{0}, x_{0}\right)$ has a nonzero limit, then the Nevai condition holds, and so links this to recent work on that question. Thus, for those interested in ergodic Schrödinger operators, by the end of this paper, we will have proven the Nevai condition in the Fibonacci model uniformly on the spectrum and for ergodic models with a.c. spectrum, Lebesgue a.e. on the essential support of the a.c. spectrum. 
We would like to thank David Damanik and Svetlana Jitomirskaya for valuable correspondence and Benjamin Weiss for valuable discussions. J.B. and B.S. would like to thank Ehud de Shalit for the hospitality of Hebrew University where some of this work was done.

\section{Subexponential Decay}

We begin with the equivalence of (1.11), (1.12), (1.13), and more:

Proposition 2.1. Let $c_{0}, c_{1}, c_{2}, \ldots$ be a sequence of nonnegative numbers and

$$
S_{n}=\sum_{j=0}^{n} c_{j}
$$

Then the following are equivalent:

$$
\begin{array}{ll}
\text { (i) } & c_{n} / S_{n} \rightarrow 0 \\
\text { (ii) } & S_{n} / S_{n+1} \rightarrow 1 \\
\text { (iii) } & c_{n+1} / S_{n} \rightarrow 0 \\
\text { (iv) } & \left(c_{n}+c_{n+1}\right) / S_{n} \rightarrow 0 \\
\text { (v) } & \left(c_{n-1}+c_{n}\right) / S_{n} \rightarrow 0
\end{array}
$$

Remarks. 1. The relevance, of course, is to

$$
c_{n}=p_{n}\left(x_{0}\right)^{2}
$$

2. If $c_{n}=e^{n^{2}}, c_{n} / S_{n} \rightarrow 1$ and $c_{n-1} / S_{n} \rightarrow 0$, so (i) is not equivalent to $c_{n-1} / S_{n} \rightarrow 0$.

Proof. (i) $\Leftrightarrow$ (ii). We have

$$
S_{n-1} / S_{n}=1-c_{n} / S_{n}
$$

So

$$
\text { (i) } \Leftrightarrow S_{n-1} / S_{n} \rightarrow 1
$$

which, by renumbering indices, is equivalent to (ii).

(ii) $\Leftrightarrow$ (iii). We have

$$
S_{n+1} / S_{n}=1+c_{n+1} / S_{n}
$$

from which (ii) is equivalent to (iii).

$$
\underline{\text { (iv) or }(\mathrm{v}) \Rightarrow(\mathrm{i})} \text {. Immediate, since for } j= \pm 1 \text {, }
$$

$$
0 \leq c_{n} / S_{n} \leq\left(c_{n}+c_{n+j}\right) / S_{n}
$$

$\underline{\text { (i) } \Rightarrow \text { (iv). }}$ Immediate from (i) $\Rightarrow$ (iii).

$(\mathrm{i}) \Rightarrow(\mathrm{v})$. Since $(\mathrm{i}) \Rightarrow S_{n-1} / S_{n} \rightarrow 1,(\mathrm{i}) \Rightarrow c_{n-1} / S_{n} \rightarrow 0$, from which (v) is immediate. 
Theorem $2.2(\Rightarrow$ Theorem 1.2). Suppose that (1.14) holds. Nevai's condition is equivalent to

$$
\int\left(x-x_{0}\right)^{2} d \eta_{n}^{\left(x_{0}\right)}(x) \rightarrow 0
$$

and (2.12) holds if and only if (1.11) holds.

Proof. By (1.8), (1.3), and the orthogonality of $p_{n}$ to $p_{n+1}$,

$$
\int\left(x-x_{0}\right)^{2} d \eta_{n}^{\left(x_{0}\right)}(x)=\frac{a_{n+1}^{2}\left[p_{n}\left(x_{0}\right)^{2}+p_{n+1}\left(x_{0}\right)^{2}\right]}{K_{n}\left(x_{0}, x_{0}\right)}
$$

By (1.14) and (i) $\Leftrightarrow$ (iv) in Proposition 2.1,

$$
\text { (2.12) } \Leftrightarrow \frac{p_{n}\left(x_{0}\right)^{2}+p_{n+1}\left(x_{0}\right)^{2}}{K_{n}\left(x_{0}, x_{0}\right)} \rightarrow 0 \Leftrightarrow \text { (1.11) }
$$

For measures, $\left\{\nu_{n}\right\}$, all supported in a fixed compact, $\nu_{n} \stackrel{w}{\longrightarrow} \delta_{x_{0}} \Leftrightarrow$ $\int\left(x-x_{0}\right)^{2} d \nu_{n} \rightarrow 0$.

In the example in Remark 2 after Proposition 2.1, $\left(c_{n-2}+c_{n-1}\right) / S_{n} \rightarrow$ 0 but (2.2) fails. However, this cannot happen for the case $c_{n}=p_{n}\left(x_{0}\right)$ : Note that if (1.14) holds,

$$
\left|p_{n}\left(x_{0}\right)\right| \leq A_{-}^{-1}\left[A_{+}+\left|x_{0}\right|+\sup _{n}\left|b_{n}\right|\right]\left[\left|p_{n-2}\left(x_{0}\right)\right|+\left|p_{n-1}\left(x_{0}\right)\right|\right]
$$

since

$$
p_{n}\left(x_{0}\right)=a_{n}^{-1}\left(\left(x_{0}-b_{n}\right) p_{n-1}\left(x_{0}\right)-a_{n-1} p_{n-2}\left(x_{0}\right)\right)
$$

and note the following obvious fact:

Proposition 2.3. Under the hypotheses and notation of Proposition 2.1, if there is a constant $K$ so that

$$
c_{n} \leq K\left(c_{n-2}+c_{n-1}\right)
$$

then (2.2) is equivalent to

$$
\frac{c_{n-2}+c_{n-1}}{S_{n}} \rightarrow 0
$$

\section{Positive Lyapunov Exponent}

We begin by proving a contrapositive of Theorem 1.3:

Proposition 3.1. If (1.11) holds, then

$$
\limsup _{n \rightarrow \infty} K_{n}\left(x_{0}, x_{0}\right)^{1 / n} \leq 1
$$

So, in particular,

$$
\limsup _{n \rightarrow \infty}\left(\left|p_{n}\left(x_{0}\right)\right|^{2}+\left|p_{n+1}\left(x_{0}\right)\right|^{2}\right)^{1 / n} \leq 1
$$


Proof. Given $\varepsilon$, pick $N$ so for $n \geq N$,

$$
\frac{\left|p_{n}\left(x_{0}\right)\right|^{2}}{K_{n}\left(x_{0}, x_{0}\right)} \leq \varepsilon
$$

Then

$$
\frac{K_{n-1}\left(x_{0}, x_{0}\right)}{K_{n}\left(x_{0}, x_{0}\right)} \geq 1-\varepsilon
$$

so, for $n \geq N$,

$$
K_{n}\left(x_{0}, x_{0}\right) \leq(1-\varepsilon)^{-1} K_{n-1}\left(x_{0}, x_{0}\right)
$$

which implies

$$
\limsup K_{n}\left(x_{0}, x_{0}\right)^{1 / n} \leq(1-\varepsilon)^{-1}
$$

Since $\varepsilon$ is arbitrary, (3.1) holds. Thus, since

$$
\left(\left|p_{n}\left(x_{0}\right)\right|^{2}+\left|p_{n+1}\left(x_{0}\right)\right|^{2}\right)^{1 / n} \leq K_{n+1}\left(x_{0}, x_{0}\right)^{1 / n}
$$

we get (3.2).

Theorem 3.2 (三 Theorem 1.3) . (1.15) $\Rightarrow$ not (1.11).

Proof. As noted, this is a contrapositive of the last statement in Proposition 3.1

Recall that the transfer matrix at $x_{0}$ is defined by

$$
\begin{aligned}
& T_{n}\left(x_{0}\right)=A_{n}\left(x_{0}\right) \ldots A_{1}\left(x_{0}\right) \\
& A_{j}\left(x_{0}\right)=\frac{1}{a_{j}}\left(\begin{array}{cc}
x_{0}-b_{j} & -1 \\
a_{j}^{2} & 0
\end{array}\right)
\end{aligned}
$$

so $\operatorname{det}\left(A_{j}\right)=\operatorname{det}\left(T_{n}\right)=1$ and

$$
\left(\begin{array}{c}
p_{n}\left(x_{0}\right) \\
a_{n} p_{n-1}\left(x_{0}\right)
\end{array}\right)=T_{n}\left(x_{0}\right)\left(\begin{array}{l}
1 \\
0
\end{array}\right)
$$

Recall also that one says the Lyapunov exponent exists if

$$
\gamma\left(x_{0}\right)=\lim _{n \rightarrow \infty} \frac{1}{n} \log \left\|T_{n}\left(x_{0}\right)\right\|
$$

exists. The Ruelle-Osceledec theorem (see, e.g., [38, Thm. 10.5.29]) says that if $\gamma\left(x_{0}\right)>0$, then there is a one-dimensional subspace, $V$, of $\mathbb{C}^{2}$, so $u \in V \backslash\{0\}$ implies

$$
\lim _{n \rightarrow \infty}\left\|T_{n}\left(x_{0}\right) u\right\|^{1 / n}=e^{-\gamma\left(x_{0}\right)}
$$

and if $u \in \mathbb{C}^{2} \backslash V$, then

$$
\lim _{n \rightarrow \infty}\left\|T_{n}\left(x_{0}\right) u\right\|^{1 / n}=e^{\gamma\left(x_{0}\right)}
$$

Thus, 
Corollary 3.3. Let (1.14) hold. If $\gamma\left(x_{0}\right)>0$, then either $x_{0}$ is a pure point of $d \rho$ or else (1.11) fails.

Proof. If (3.12) holds for $u=\left(\begin{array}{l}1 \\ 0\end{array}\right)$, then by (3.10), $\left|p_{n}\left(x_{0}\right)\right| \leq C e^{-\gamma\left(x_{0}\right) n / 2}$ so $p_{n} \in \ell^{2}$ and $x_{0}$ is a pure point. So if $x_{0}$ is not a pure point, then (3.13) holds, so since $A_{-}>0$, (3.13) implies

$$
\lim _{n \rightarrow \infty}\left(\left|p_{n}\left(x_{0}\right)\right|^{2}+\left|p_{n+1}\left(x_{0}\right)\right|^{2}\right)^{1 / n}=e^{\gamma\left(x_{0}\right)}>1
$$

and so (1.11) fails.

Example 3.4 (Szwarc [46]). This example is mainly of historical interest. This is a modification of [46], but uses the key notion of having an isolated point of $\sigma_{\text {ess }}(J)$, which is not an eigenvalue. We begin by noting that the whole-line Jacobi matrix, $J_{\infty}$, with $a_{n} \equiv 1, b_{j}=0(j \neq 0)$ and $b_{0}=\frac{3}{2}$ has $E=\frac{5}{2}\left(=2+\frac{1}{2}\right)$ as an eigenvalue with eigenfunction $\left(\frac{1}{2}\right)^{|n|}$, so $\sigma\left(J_{\infty}\right)=[-2,2] \cup\left\{\frac{5}{2}\right\}$.

Now let $J$ be the one-sided Jacobi matrix with $a_{n} \equiv 1$ and

$$
b_{n}= \begin{cases}\beta & n=1 \\ \frac{3}{2} & n=k^{2}, k=2,3, \ldots \\ 0 & n \neq k^{2} \text { all } k\end{cases}
$$

where $\beta$ will be adjusted below.

Standard right-limit theorems (see, e.g., [22]) imply

$$
\sigma_{\text {ess }}(J)=[-2,2] \cup\left\{\frac{5}{2}\right\}
$$

so, in particular, $\frac{5}{2} \in \sigma(J)$. Moreover, since the nonzero $b_{n}$ 's are of zero density, it is easy to see that

$$
\begin{aligned}
\gamma\left(\frac{5}{2}\right) & =\log \left(\text { spectral radius of }\left(\begin{array}{cc}
\frac{5}{2} & -1 \\
1 & 0
\end{array}\right)\right) \\
& =\log (2)
\end{aligned}
$$

since $\left(\begin{array}{cc}\frac{5}{2} & -1 \\ 1 & 0\end{array}\right)$ has eigenvalues 2 and $\frac{1}{2}$.

It is easy to see that there is exactly one choice of $\beta$ for which $\frac{5}{2}$ is an eigenvalue of $J$. For any other choice, Corollary 3.3 is applicable, and so the Nevai condition fails at $\frac{5}{2} \in \sigma(J)$.

Example 3.5. Let $a_{n} \equiv \frac{1}{2}$ and $b_{n}(\omega)$ be i.i.d. random variables uniformly distributed in $[-1,1]$. This is an Anderson model for which it is well-known that for a.e. choice of $\omega$ (see, e.g., [7]), the associated measure $d \rho_{\omega}$ has pure point spectrum filling $[-2,2]$. Moreover, for a.e. $\omega$ and quasi-every $x_{0} \in[-2,2]$, one has Lyapunov exponent $\gamma\left(x_{0}\right)>0$. Since the set of eigenvalues is countable, for quasi-every $x_{0} \in[-2,2]$, 
Corollary 3.3 is applicable. Thus, $\sigma\left(J_{\omega}\right)=[-2,2]$, but for quasi-every $x_{0} \in[-2,2]$, the Nevai condition fails.

\section{A Regular Measure for Which the Nevai Condition Fails on a Set of Positive Lebesgue Measure}

Example 4.1. Let $J$ be a Jacobi matrix with $b_{n} \equiv 0$ and $a_{n}$ described as follows. Partition $\{1,2, \ldots\}$ into successive blocks $A_{1}, C_{1}, A_{2}, C_{2} \ldots$, where

$$
\#\left(A_{j}\right)=3^{j^{2}} \quad \#\left(C_{j}\right)=2^{j^{2}}
$$

On $A_{j}, a_{n} \equiv 1$ and on $C_{j}, a_{n} \equiv \frac{1}{2}$. Since the $3^{j^{2}}$ blocks are much larger than the $2^{j^{2}}$ blocks,

$$
\lim _{n \rightarrow \infty}\left(a_{1} \ldots a_{n}\right)^{1 / n}=1
$$

and since $\left|a_{n}\right| \leq 1,\|J\| \leq 2$ so $\sigma(J) \subset[-2,2]$. By a simple trial function argument, $[-2,2] \subset \sigma(J)$. Thus, $J$ is regular for $[-2,2]$, but, of course, not in Nevai class since $a_{n} \nrightarrow 1$.

We will prove that

Theorem 4.2. For Lebesgue a.e. $x_{0} \in[-2,2] \backslash[-1,1]$, the Nevai condition fails.

The intuition, which we will implement, goes as follows: For $x_{0}$ in the specified set, in $A_{j}$ regions, solutions of the eigenfunction equation are linear combinations of plane waves. So in such regions, $p_{n}\left(x_{0}\right)$ hardly grows or decays. In $C_{j}$ regions, they are linear combinations of growing and decaying exponentials, so usually, the growing exponentials will win and the $p_{n}\left(x_{0}\right)$ will grow exponentially. $C_{j+1}$ is much bigger than $C_{j}$ (indeed, $\left.\#\left(C_{j+1}\right)=24^{j} \#\left(C_{j}\right)\right)$, so at the center of $C_{j+1},\left|p_{n}\left(x_{0}\right)\right|^{2}$ will be much bigger than $\sum_{k \in \cup_{\ell=1}^{j}\left(A_{\ell} \cup C_{\ell}\right) \cup A_{j+1}}\left|p_{k}\left(x_{0}\right)\right|^{2}$ and comparable to $\sum_{\substack{k \in C_{j+1} \\ k \leq n-1}}\left|p_{k}\left(x_{0}\right)\right|^{2}$, which will prevent (1.11) from holding.

We will say more about the general strategy shortly, but we first implement the initial step:

Proposition 4.3. Suppose for a given $x_{0}$, there are $C, D$ and $\alpha, \beta>0$ so that

(i) For all $j$ and $n \in C_{j} \cup A_{j+1}$,

$$
\left|p_{n}\left(x_{0}\right)\right| \leq C^{j+1} \exp \left(\alpha 2^{j^{2}}\right)
$$

(ii) For all large $j$ and $n_{j}$, the "center" of $C_{j}$, we have

$$
\left(\left|p_{n_{j}-1}\left(x_{0}\right)\right|^{2}+\left|p_{n_{j}}\left(x_{0}\right)\right|^{2}\right)^{1 / 2} \geq D^{-(j+1)} \exp \left(\beta 2^{j^{2}}\right)
$$

Then (1.11) (and so the Nevai condition) fails at $x_{0}$. 
Remarks. 1. In fact, the proof shows that (4.4) must only hold for infinitely many $j$ 's.

2. Since \# $\left(C_{j}\right)$ is even, it does not have a strict center. By "center," we mean one half unit prior to the midpoint.

Proof. Suppose (1.11) holds. Then, since (1.13) holds, for any $\varepsilon>0$, there exists $N(\varepsilon)$, so for $n \geq N(\varepsilon)$,

$$
\left|p_{n+1}\left(x_{0}\right)\right|^{2} \leq \varepsilon \sum_{k=1}^{n}\left|p_{k}\left(x_{0}\right)\right|^{2}
$$

SO

$$
\sum_{k=1}^{n+1}\left|p_{k}\left(x_{0}\right)\right|^{2} \leq(1+\varepsilon) \sum_{k=1}^{n}\left|p_{k}\left(x_{0}\right)\right|^{2}
$$

and thus, for $n \geq m \geq N(\varepsilon)$,

$$
\left|p_{n-1}\left(x_{0}\right)\right|^{2}+\left|p_{n}\left(x_{0}\right)\right|^{2} \leq(1+\varepsilon)^{n-m} \sum_{k=1}^{m}\left|p_{k}\left(x_{0}\right)\right|^{2}
$$

Now, suppose $C_{j}$ is such that its leftmost point, $m_{j}+1$, has $m_{j} \geq$ $N(\varepsilon)$ and let $n_{j}$ be the center of $C_{j}$. Then by (4.3),

$$
\sum_{k=1}^{m_{j}}\left|p_{k}\left(x_{0}\right)\right|^{2} \leq C^{2 j} \exp \left(2 \alpha 2^{(j-1)^{2}}\right)\left[\sum_{k=1}^{j} 2^{k^{2}}+3^{k^{2}}\right]
$$

and by (4.4),

$$
\left|p_{n_{j}-1}\left(x_{0}\right)\right|^{2}+\left|p_{n_{j}}\left(x_{0}\right)\right|^{2} \geq D^{-2(j+1)} \exp \left(2 \beta 2^{j^{2}}\right)
$$

Pick $\varepsilon$ so $(1+\varepsilon) \leq e^{2 \beta}$. Since $n_{j}-m_{j}=\frac{1}{2}\left(2^{j^{2}}\right)$, (4.7) says that so long as $m \geq N(\varepsilon)$,

$$
D^{-2(j+1)} \exp \left(2 \beta 2^{j^{2}}\right) \leq \exp \left(\beta 2^{j^{2}}\right) C^{2 j} \exp \left(2 \alpha 2^{(j-1)^{2}}\right)\left(2 j 3^{j^{2}}\right)
$$

Since $2 \beta>\beta+44^{-j} \alpha$ for $j$ large, (4.10) cannot hold for large $j$. This contradiction implies that (4.5) cannot hold for this value of $\varepsilon$, and thus, (1.11) fails.

The upper bound, (4.3), will be easy from transfer matrix arguments. The lower bound, (4.4), is much more subtle. Indeed, it implies that for those $n$ 's, asymptotically $\left|p_{n}\left(x_{0}\right)\right| \geq n^{\ell}$ for all $\ell$. On the other hand, for a.e. $x_{0}$ with respect to a spectral measure, $p_{n}\left(x_{0}\right)$ is polynomially bounded. Thus, (4.3) must fail for a dense set of $x_{0}$ 's! Fortunately, this kind of problem has been faced before in spectral theory contexts and we will be able to borrow a technique from Jitomirskaya-Last [17]. We turn first to the upper bound. 
For $x_{0} \in(-2,2) \backslash[-1,1]$, define $\theta\left(x_{0}\right), \eta\left(x_{0}\right)$ by

$$
2 \cos \theta\left(x_{0}\right)=x_{0} \quad \cosh \eta\left(x_{0}\right)=\left|x_{0}\right|
$$

Let $Q\left(x_{0}\right), R\left(x_{0}\right)$ be the one-unit transfer matrices for $b_{n} \equiv 0, a_{n} \equiv 1$ and $b_{n} \equiv 0, a_{n} \equiv \frac{1}{2}$. Then $Q\left(x_{0}\right)$ has $e^{ \pm i \theta_{0}}$ as eigenvalues and $R\left(x_{0}\right)$ has eigenvalues $e^{ \pm \eta\left(x_{0}\right)}$ if $x_{0}>0$ and $-e^{ \pm \eta\left(x_{0}\right)}$ if $x_{0}<0$. It follows that for some constants $c\left(x_{0}\right), d\left(x_{0}\right)$,

$$
\begin{aligned}
\left\|Q\left(x_{0}\right)^{k}\right\| & \leq c\left(x_{0}\right) \\
\left\|R\left(x_{0}\right)^{k}\right\| & \leq d\left(x_{0}\right) e^{k \eta\left(x_{0}\right)}
\end{aligned}
$$

with $c, d$ bounded uniformly on compacts of $(-2,2) \backslash[-1,1]$. This lets us prove that

Proposition 4.4. For any compact subset $K$ of $(-2,2) \backslash[-1,1]$, there are constants $C, \alpha$ so that for all $n, j$ and $x_{0} \in K$ with $n \in C_{j} \cup A_{j+1}$, we have

$$
\left\|T_{n}\left(x_{0}\right)\right\| \leq C^{j+1} \exp \left(\alpha 2^{j^{2}}\right)
$$

Remark. Since $p_{n}\left(x_{0}\right)$ is a matrix element of $T_{n}$, we immediately have (4.3).

Proof. For $n \in A_{j+1}, T_{n}\left(x_{0}\right)$ is a product of $(j+1)$ factors of products of $Q\left(x_{0}\right)$ and $j$ factors of products of $R\left(x_{0}\right)$. Thus, by (4.12) and (4.13),

$$
\left\|T\left(x_{0}\right)\right\| \leq c\left(x_{0}\right)^{j+1} d\left(x_{0}\right)^{j} \exp \left(\eta\left(x_{0}\right) \sum_{\ell=1}^{j} 2^{\ell^{2}}\right)
$$

For $n \in C_{j}$, the estimate is similar, but $c\left(x_{0}\right)^{j+1}$ is replaced by $c\left(x_{0}\right)^{j}$ and $\sum_{\ell=1}^{j} 2^{\ell^{2}}$ by a smaller sum.

Since $c, d$, and $\eta$ are bounded on $K$ and

$$
\sum_{\ell=1}^{j} 2^{\ell^{2}} \leq 2^{\ell^{2}}\left[1+\frac{1}{2}+\frac{1}{4}+\ldots\right]=22^{\ell^{2}}
$$

we obtain (4.14).

To get the lower bound following Jitomirskaya-Last [17], we need to consider Weyl solutions and Green's functions. For $\operatorname{Im} z>0$, there is a unique solution $u_{n}^{+}(z)$ of

$$
a_{n} u_{n+1}+\left(b_{n}-z\right) u_{n}+a_{n-1} u_{n-1}=0
$$

defined for $n=1,2, \ldots$ (with $a_{0} \equiv 1$ ) which is $\ell^{2}$ at infinity and normalized by

$$
u_{0}^{+}(z)=-1
$$


This is the Weyl solution. The spectral theorist's Green's function (different from the Green's function of potential theory!) is defined for $n, m \geq 1$ by

$$
G_{n m}(z)=\left\langle\delta_{n},(J-z)^{-1} \delta_{m}\right\rangle
$$

Then $G_{n m}=G_{m n}$ and, for $1 \leq n \leq m$,

$$
G_{n m}(z)=p_{n-1}(z) u_{m}^{+}(z)
$$

As usual, the Wronskian is constant and, by $a_{0} \equiv 1$ and (4.18) plus $p_{-1}=0, p_{0}=1$, this constant is 1 . So for all $n=0,1,2, \ldots$,

$$
a_{n}\left(u_{n+1}^{+}(z) p_{n-1}(z)-u_{n}^{+}(z) p_{n}(z)\right)=1
$$

By (4.19), $G_{n m}$ is the Borel transform of a signed measure, and so for Lebesgue a.e. $x_{0}$, it has boundary values $G_{n m}\left(x_{0}+i 0\right)$. In particular, since $p_{0}=1, u_{n}^{+}=G_{1 n}$ has a.e. boundary values, $u_{n}^{+}\left(x_{0}+i 0\right)$. (4.20) and (4.21) still hold for $z=x_{0}+i 0$.

In particular, for our example where $a_{n}$ is 1 or $\frac{1}{2}$, (4.21) and the Schwarz inequality imply that

$$
\left(p_{n}\left(x_{0}\right)^{2}+p_{n-1}\left(x_{0}\right)^{2}\right) \geq\left(\left|u_{n+1}^{+}\left(x_{0}+i 0\right)\right|^{2}+\left|u_{n}^{+}\left(x_{0}+i 0\right)\right|^{2}\right)^{-1}
$$

So to get an exponentially growing lower bound on $p_{n}\left(x_{0}\right)^{2}+p_{n-1}\left(x_{0}\right)^{2}$, we only need to get an exponentially decaying upper bound on $\mid u_{n}^{+}\left(x_{0}+\right.$ $i 0) \mid$ and $\left|u_{n+1}^{+}\left(x_{0}+i 0\right)\right|$.

Now fix $1<k<\ell<\infty$ and define $\tilde{J}$ to be the Jacobi matrix obtained by replacing $a_{k}$ and $a_{\ell}$ by 0 . Thus, under

$$
\begin{gathered}
\ell^{2}(\{1,2, \ldots\})=\ell^{2}(\{1, \ldots, k\}) \oplus \ell^{2}(\{k+1, \ldots, \ell\}) \\
\oplus \ell^{2}(\{\ell+1, \ell+2, \ldots,\}) \\
\tilde{J}=J_{\mathrm{L}} \oplus J_{\mathrm{M}} \oplus J_{\mathrm{R}}
\end{gathered}
$$

(L, M, R for left, middle, right).

Proposition 4.5. Let $\widetilde{G}$ be the Green's function for $\tilde{J}$. Let

$$
k+1 \leq n \leq \ell
$$

Then

$$
G_{1 n}=-a_{k} G_{1 k} \widetilde{G}_{k+1 n}-a_{\ell} G_{1 \ell+1} \widetilde{G}_{\ell n}
$$

Proof. Define

$$
\Gamma=J-\tilde{J}
$$

Then

$$
(J-z)^{-1}=(\tilde{J}-z)^{-1}-(J-z)^{-1} \Gamma(\tilde{J}-z)^{-1}
$$

SO

$$
G_{1 n}=\widetilde{G}_{1 n}-\sum_{m, r} G_{1 m} \Gamma_{m r} \widetilde{G}_{r n}
$$


Since 1 and $n$ lie in different blocks in the direct sum (4.24), $\widetilde{G}_{1 n}=0$. $\Gamma$ is a rank four operator; since $\widetilde{G}_{k n}=\widetilde{G}_{\ell+1 n}=0$, two terms in the sum in (4.29) vanish. The result is (4.26).

Proposition 4.6. Let $J^{(k)}$ be the $k \times k$ matrix with 0 's on the diagonal and $\frac{1}{2}$ in each of the two principal off-diagonals. Let $G^{(k)}(z)$ be the matrix $\left(J^{(k)}-z\right)^{-1}$. Then for $x_{0} \notin[-1,1]$ and $m \leq n$,

$$
G_{m n}^{(k)}\left(x_{0}\right)=\frac{2\left(w^{-m}-w^{m}\right)\left(w^{-(k+1-n)}-w^{(k+1-n)}\right)}{\left(w^{-1}-w\right)\left(w^{-(k+1)}-w^{(k+1)}\right)}
$$

where $|w|>1$ and solves

$$
w^{-1}+w=2 x_{0}
$$

In particular, for any compact $K \in(-2,2) \backslash[-1,1]$, there are $\gamma>0$ and $C$ so that for all $x_{0} \in K$ and all $k=2 r$,

$$
\left|G_{1 n}^{(2 r)}\left(x_{0}\right)\right| \leq C e^{-\gamma r}
$$

for

$$
n=r, r-1
$$

Proof. $w^{n}$ and $w^{-n}$ solve

$$
\frac{1}{2}\left(u_{n+1}+u_{n-1}\right)=x_{0} u_{n}
$$

so $w^{-n}-w^{n}$ solves (4.34) with $u_{0}=0$ boundary condition, while $w^{-(k+1-n)}-w^{(k+1-n)}$ solves it with $u_{k+1}=0$ boundary condition. The numerator in (4.30) is twice their product and the denominator twice their Wronskian, proving (4.30). (4.32) follows by noting that the dominant term in the numerator is $w^{r}$, while in the denominator, $w^{2 r}$.

Thus, in (4.26), where $k+1$ and $\ell$ are taken to be the edge of a $C_{j}$ block and $n$ to be the center or one less, the $\widetilde{G}$ terms are exponentially small. We thus need estimates on the set of $x_{0}$ for which $G_{1 k}\left(x_{0}\right)$ can be large. Here we recall Loomis's theorem:

Theorem 4.7 (Loomis [23]). Let $\mu$ be a complex measure on $\mathbb{R}$ of finite total variation $\|\mu\|$. Let

$$
F_{\mu}(x)=\lim _{\varepsilon \downarrow 0} \int \frac{d \mu(y)}{y-(x+i \varepsilon)}
$$

which exists for Lebesgue a.e. $x$. Then with $|\cdot|=$ Lebesgue measure,

$$
\left|\left\{x|| F_{\mu}(x) \mid>M\right\}\right| \leq \frac{C\|\mu\|}{M}
$$

for a universal constant $C$. 
Remarks. 1. The history is complicated and is partly described in Loomis [23]. The result for measures $\mu$ which are absolutely continuous is due earlier to Kolmogorov.

2. For $d \mu=f(x) d x$ with $f \in L^{1}$, the optimal constant was found by Davis [12]. For purely singular positive measures, the result is essentially due to Boole [2] with constant $C=2$, which is optimal-indeed, one has equality.

3. In [17], they only used Boole's equality since their measure is purely singular. That is true here also, but not in the next section. In any event, it is useful to know pure singularity is not needed a priori, although it follows on $[-2,2] \backslash[-1,1]$ from the estimates here.

Proposition 4.8. Let $n_{1}<n_{2}<\ldots$ be an arbitrary sequence of indices. Let $\delta>0$. Then for a.e. $x_{0} \in \mathbb{R}, \exists J\left(x_{0}\right)$ so that

$$
j>J\left(x_{0}\right) \Rightarrow\left|G_{1 n_{j}}\left(x_{0}+i 0\right)\right| \leq e^{\delta j}
$$

Proof. This is a standard Borel-Cantelli argument. Let $\chi_{j}$ be the characteristic function of $\left\{x_{0}|| G_{1 n_{j}}\left(x_{0}\right) \mid>e^{\delta j}\right\}$. Since $G_{1 n_{j}}\left(x_{0}+i 0\right)$ is of the form (4.35) for a measure of variation at most 1 ,

$$
\int \chi_{j}(x) d x \leq C e^{-\delta j}
$$

Thus,

$$
\int \sum_{j=1}^{\infty} \chi_{j}(x) d x<\infty
$$

which implies that for a.e. $x_{0}, \sum_{j=1}^{\infty} \chi_{j}\left(x_{0}\right)<\infty$. Since each $\chi_{j}\left(x_{0}\right)$ is 0 or 1 , only finitely many are nonzero, that is, for all large $j$, (4.37) holds.

Proof of Theorem 4.2. By Propositions 4.3 and 4.4, we only need to prove that (4.4) holds for Lebesgue a.e. $x_{0}$. By (4.22), it suffices to prove exponentially decaying upper bounds on $u_{n_{j}}^{+}, u_{n_{j}+1}^{+}$(for the same $n_{j}$ as (4.4)). As noted, $u_{n}^{+}=G_{1 n}$, so it suffices to prove exponentially decaying upper bounds on $G_{1 n_{j}}, G_{1 n_{j}+1}$.

We use (4.26) with $k, \ell$ the lower and upper edge of the $C_{j}$ block. By Proposition 4.6,

$$
\left|\widetilde{G}_{k+1 n_{j}}\right|+\left|\widetilde{G}_{k+1 n_{j}+1}\right|+\left|\widetilde{G}_{\ell n_{j}}\right|+\left|\widetilde{G}_{\ell n_{j}+1}\right| \leq C \exp \left(-\gamma 2^{j^{2}}\right)
$$

for some $\gamma>0$.

Thus, the result follows from Proposition 4.8, for eventually each of the complementary Green's functions in (4.26) is bounded by $\exp \left(\frac{1}{2} \gamma j\right) \leq \exp \left(\frac{1}{2} \gamma 2^{j^{2}}\right)$ and (4.4) holds with $\beta=\frac{1}{2} \gamma$. 
Finally, we want to note that $J$ has a two-sided right limit which has $a_{n}=\frac{1}{2}$ for $n \leq-1$ and $a_{n}=1$ for $n \geq 0$. There is no set of positive Lebesgue measure on which $J$ is reflectionless, so, by a theorem of Remling [36], $J$ has purely singular spectrum.

\section{A Regular Measure With Some A.C. Spectrum}

As we noted at the end of the last section, Example 4.1 has no a.c. spectrum. Of course, if Conjecture 1.4 is true, then Lebesgue a.e. on the a.c. spectrum, the Nevai condition holds. So an example like Example 4.1 cannot have a.c. spectrum on $[-2,2] \backslash[-1,1]$ but it can on $[-1,1]$. The example in this section shows that a.c. spectrum is indeed possible on $[-1,1]$.

Example 5.1. Let $J$ be a Jacobi matrix with $b_{n} \equiv 0$ and $a_{n}$ described as follows: Partition $\{1,2, \ldots\}$ into successive blocks $A_{1}, B_{1}, C_{1}, D_{1}, A_{2}, B_{2}, \ldots$, where

$$
\#\left(A_{j}\right)=3^{j^{2}} \quad \#\left(C_{j}\right)=2^{j^{2}} \quad \#\left(B_{j}\right)=\#\left(D_{j}\right)=j^{6}-1
$$

On $A_{j}, a_{n} \equiv 1$, on $C_{j}, a_{n} \equiv \frac{1}{2}$, and on $B_{j}$ and $D_{j}, \log \left(a_{n}^{2}\right)$ linearly interpolates from $\log \left(\frac{1}{4}\right)$ to $\log (1)$, that is, for $n \in B_{j}$,

$$
\frac{a_{n}^{2}}{a_{n-1}^{2}}=c_{j}
$$

and for $n \in D_{j}$,

$$
\frac{a_{n-1}^{2}}{a_{n}^{2}}=c_{j}
$$

where

$$
c_{j}^{j^{6}}=\frac{1}{4}
$$

so that

$$
1-c_{j}=k j^{-6}+o\left(j^{-6}\right)
$$

for a suitable nonzero constant $k$. In particular,

$$
\left|1-c_{j}\right| \leq E_{0} j^{-6}
$$

for some $E_{0}$.

As in Example 4.1, this $J$ is regular with spectrum $[-2,2]$. We will prove that

Theorem 5.2. On $[-2,2] \backslash[-1,1], J$ has purely singular spectrum and for Lebesgue a.e. $x_{0}$ in this set, the Nevai condition fails. On $(-1,1)$, $J$ has purely a.c. spectrum and for all $x_{0} \in(-1,1)$, the Nevai condition holds uniformly on compact subsets.

The technical key to the new elements of this example is 
Theorem 5.3. For $x_{0} \in(-1,1)$, let $u_{n}\left(x_{0}, \theta_{0}\right)$ be the solution of (4.17) (with $a_{0} \equiv 1$ and $z=x_{0}$ ) for $n=1,2, \ldots$, with

$$
u_{0}=\cos \theta_{0} \quad u_{1}=\sin \theta_{0}
$$

Then for any compact set $K \subset(-1,1)$, there is a constant, $C$, so that for all $x_{0} \in K$, all $\theta_{0}$ and all $n$,

$$
\left|u_{n}\left(x_{0}, \theta_{0}\right)\right| \leq C
$$

Proof of Theorem 5.2 given Theorem 5.3. $J$ has as one of its right limits, $J_{r}$, the two-sided matrix with $b_{n} \equiv 0, a_{n} \equiv \frac{1}{2}$ whose a.c. spectrum is $\Sigma_{\mathrm{ac}}\left(J_{r}\right)=[-1,1]$. By a theorem of Last-Simon [21], $\Sigma_{\mathrm{ac}}(J) \subset[-1,1]$, so $J$ has purely singular spectrum on $[-2,2] \backslash[-1,1]$. The results on this set for the Nevai condition follow the arguments in Section 4 without change.

Theorem 5.3 implies that the transfer matrix $T_{n}\left(x_{0}\right)$ is uniformly bounded in $n$ and $x_{0} \in K \subset(-1,1)$ compact. Carmona's formula (see, e.g., [38, Thm. 10.7.5]; also [4, 21, 37, 40]) then implies the spectrum is purely a.c. on $(-1,1)$.

A bounded transfer matrix also implies $p_{n}\left(x_{0}\right)^{2}$ bounded above, and given constancy of the Wronskian (i.e., $\operatorname{det}\left(T_{n}\right)=1$ ), uniform lower bounds on $p_{n}\left(x_{0}\right)^{2}+p_{n+1}\left(x_{0}\right)^{2}$. Thus, on $(-1,1)$,

$$
\frac{p_{n}\left(x_{0}\right)^{2}}{\sum_{j=0}^{n} p_{j}\left(x_{0}\right)^{2}} \leq \frac{C}{n} \rightarrow 0
$$

proving (1.11).

The situation we need to control for Theorem 5.3 has much in common with those studied by Kiselev-Last-Simon [19] and their techniques will work here. We note that in our situation, $\sum_{n}\left(a_{n+1}-a_{n}\right)^{2}+$ $\left(b_{n+1}-b_{n}\right)^{2}<\infty$, a general condition studied recently by Denisov [13], but under the additional assumptions that $a_{n} \equiv 1, b_{n} \rightarrow 0$. It would be interesting to see if one can extend his ideas to this context (see Conjecture 9.5 and the discussion following it below).

We depend on the EFGP transform, as do [19, but we need to allow modification for our case where $a_{n}$ is not identically one, as it is in [19]. Since $b_{n} \equiv 0$ for us, we state the equations for that case. One defines $R_{n}, \theta_{n}$ by

$$
\begin{aligned}
& R_{n} \sin \left(\theta_{n}\right)=a_{n} u_{n} \sin \left(k_{n}\right) \\
& R_{n} \cos \left(\theta_{n}\right)=a_{n}\left(u_{n+1}-u_{n} \cos \left(k_{n}\right)\right)
\end{aligned}
$$

where $k_{n}$ is given by

$$
2 \cos \left(k_{n}\right)=\frac{x_{0}}{a_{n}}
$$


We note, since $a_{n} \geq \frac{1}{2}$ and $\sup _{x_{0} \in K}\left|x_{0}\right|<1$, that uniformly for $x_{0} \in K$ and all $n$,

$$
\varepsilon \leq k_{n} \leq \pi-\varepsilon
$$

for some $\varepsilon>0$ (depending on $K$ ).

As in [19], straightforward manipulations of the eigenfunction equation show (4.17) is equivalent to

$$
\begin{aligned}
\frac{R_{n+1}^{2}}{R_{n}^{2}} & =1+\left(a_{n+1}^{2}-a_{n}^{2}\right) \frac{\sin ^{2}\left(\theta_{n}+k_{n}\right)}{a_{n}^{2} \sin ^{2}\left(k_{n}\right)} \\
\cot \left(\theta_{n+1}\right) & =\frac{a_{n}}{a_{n+1}} \frac{\sin \left(k_{n}\right)}{\sin \left(k_{n+1}\right)} \cot \left(\theta_{n}+k_{n}\right)
\end{aligned}
$$

$R_{1}$ and $\theta_{1}$ are functions of $\theta_{0}$ (given $a_{1}=a_{2}=1$ ) and $R_{1}$ is, for $x_{0} \in K$, uniformly bounded above and below. Moreover, by (5.10) and (5.13), for $C$ depending only on $K$,

$$
\left|u_{n}\right| \leq C R_{n}
$$

Define

$$
X_{n}=\frac{\left(a_{n+1}^{2}-a_{n}^{2}\right) \sin ^{2}\left(\theta_{n}+k_{n}\right)}{a_{n}^{2} \sin ^{2}\left(k_{n}\right)}
$$

By Lemma 3.5 of [19] and $\sup _{n}\left|X_{n}\right|<\infty$, it suffices to prove that

$$
\sup _{N}\left|\sum_{j=1}^{N} X_{j}\right|<\infty
$$

Define $\widetilde{B}_{j}, \widetilde{D}_{j}$ by adding to $B_{j}, D_{j}$ the index one before (i.e., the top index of $A_{j}$ and $\left.C_{j}\right)$. Then $X_{n}$ is only nonzero on $\cup_{j}\left(\widetilde{B}_{j} \cup \widetilde{D}_{j}\right)$. On $\widetilde{B}_{j} \cup \widetilde{D}_{j}$, by (5.2), (5.3), and (5.6),

$$
\left|X_{n}\right| \leq E\left(x_{0}\right) j^{-6}
$$

where

$$
E\left(x_{0}\right)=E_{0} \sup _{n} \frac{1}{\sin ^{2}\left(k_{n}\right)}
$$

is bounded above on $K$ by (5.13). Theorem 5.3 is reduced to proving

$$
\sup _{N}\left|\sum_{n=1}^{N} X_{n}\right|<\infty
$$

uniformly in $\theta_{1}$ and $x_{0} \in K$.

Next, we note that one can write

$$
X_{n}=X_{n}^{\sharp}+\tilde{X}_{n}
$$

using

$$
\sin ^{2}\left(\theta_{n}+k_{n}\right)=\frac{1}{2}\left(1-\cos \left(2\left(\theta_{n}+k_{n}\right)\right)\right)
$$


The $X_{n}^{\sharp}$ terms are independent of $\theta_{n}$ and there is a symmetry between points in $\widetilde{B}_{j}$ and $\widetilde{D}_{j}$ which, given the opposite signs of $1-a_{n}^{2} / a_{n+1}^{2}$, causes a partial cancellation, that is, since $c_{j}^{-1}-c_{j}=O\left(j^{-6}\right)$,

$$
\sum_{n \in \widetilde{B}_{j}} X_{n}^{\sharp}+\sum_{n \in \widetilde{D}_{j}} X_{n}^{\sharp}=O\left(j^{-6}\right)
$$

Moreover,

$$
\sum_{n \in \widetilde{B}_{j}}\left|X_{n}^{\sharp}\right| \leq E\left(x_{0}\right)
$$

These together (plus the approximate cancellation) implies

$$
\sup _{N}\left|\sum_{n=1}^{N} X_{n}^{\sharp}\right| \leq E\left(x_{0}\right)+O\left(\sum j^{-6}\right)
$$

so we have reduced the proof of (5.21), and so of Theorem [5.3, to proving

$$
\sup _{N}\left|\sum_{n=1}^{N} \tilde{X}_{n}\right|<\infty
$$

uniformly in $\theta_{1}$ and $x_{0} \in K$.

We want to use cancellations of sums of cosines - more explicitly, that sums of $M$ cosines with suitably varying phase are of order 1 , not $M$. Here is what we need:

Lemma 5.4. For any $q \in(0,2 \pi)$, any $\theta$, and $M$,

$$
\left|\sum_{\ell=1}^{M} \cos (q \ell+\theta)\right| \leq\left[\sin \left(\frac{q}{2}\right)\right]^{-1}
$$

Proof. Since $\cos (\psi)=\operatorname{Re}\left(e^{i \psi}\right)$, it suffices to prove this if $\cos (q \ell+\theta)$ is replaced by $e^{i(q \ell+\theta)}$. By summing a geometric series,

$$
\begin{aligned}
\left|\sum_{\ell=1}^{M} e^{i(q \ell+\theta)}\right| & =\left|\frac{e^{i[(M+1) q+\theta]}-e^{i[q+\theta]}}{e^{i q}-1}\right| \\
& \leq \frac{2}{2\left|\left(e^{i q / 2}-e^{-i q / 2}\right) / 2\right|}=\frac{1}{\sin \left(\frac{q}{2}\right)}
\end{aligned}
$$

In $\cos \left(\theta_{n}+k_{n}\right)$, both $k_{n}$ and $\theta_{n}$ are $n$-dependent. But over subblocks small compared to $j^{6}, k_{n}$ is close to constant and $\theta_{n+1}-\theta_{n}$ is close to constant. Thus, we break $\widetilde{B}_{j}$ and $\widetilde{D}_{j}$ into $j^{4}$ blocks, each with $j^{2}$ 
members, call them $\left\{\widetilde{B}_{j, \ell}\right\}_{\ell=1}^{j^{4}}$ and $\left\{\widetilde{D}_{j, \ell}\right\}_{\ell=1}^{j^{4}}$. For any $n$ in some $\widetilde{B}_{j}$ or $\widetilde{D}_{j}$, let $\beta_{n}$ be the first element of the subblock containing $n$ and

$$
\kappa_{n}=k_{\beta_{n}}
$$

Clearly, with constants uniformly bounded over $K$ (below, $C$ will stand for a generic constant bounded on any compact $K \subset(-1,1)$ ),

$$
\begin{aligned}
\left|n-\beta_{n}\right| & \leq C j^{2} \\
\left|k_{n}-\kappa_{n}\right| & \leq C j^{-4}
\end{aligned}
$$

(5.30) comes from the fact that over a subblock, $a_{n}$ changes by at most $j^{2} O\left(j^{-6}\right)$.

In (5.15), the ratio of $a$ 's is 1 plus an error of order $j^{-6}$, so given that arc cot has bounded derivatives,

$$
\left|\theta_{n+1}-\left(\theta_{n}+k_{n}\right)\right| \leq C j^{-6}
$$

and so,

$$
\left|\theta_{n+1}-\theta_{n}-\kappa_{n}\right| \leq C j^{-4}
$$

This implies that if

$$
\tilde{\theta}_{n}=\theta_{\beta_{n}}+\left(n-\beta_{n}\right) \kappa_{n}
$$

then

$$
\left|\theta_{n}-\tilde{\theta}_{n}\right| \leq C j^{-2}
$$

Define $Y_{n}$ to be $\tilde{X}_{n}$ with $\cos \left(2\left(\theta_{n}+k_{n}\right)\right)$ replaced by $\cos \left(2\left(\tilde{\theta}_{n}+\kappa_{n}\right)\right)$ and $a_{n}^{2} \sin ^{2}\left(k_{n}\right)$ by $a_{\beta_{n}}^{2} \sin ^{2}\left(\kappa_{n}\right)$. By (5.30) and (5.34) (since $a_{n+1}^{2}-a_{n}^{2} \sim j^{-6}$ ), on $\widetilde{B}_{j} \cup \widetilde{D}_{j}$,

$$
\left|Y_{n}-\widetilde{X}_{n}\right| \leq C j^{-8}
$$

and

$$
\sum_{n \in \widetilde{B}_{j} \cup \widetilde{D}_{j}}\left|Y_{n}-\widetilde{X}_{n}\right| \leq C j^{-2}
$$

which is summable in $j$. Thus, to prove (5.27), we need

$$
\sup _{N}\left|\sum_{n=1}^{N} Y_{n}\right|<\infty
$$

uniformly in $\theta_{1}$ and $K$.

Proof of Theorem 5.3. As noted, we are reduced to proving (5.36). $\{1, \ldots, N\}$ can be broken into sums over 0 (i.e., $A_{j}$ and $C_{j}$, except for their final indices), sums over some number of subblocks, and one further partial subblock. Summing over a single subblock is, by Lemma 5.4 (given that, by (5.13), $2 \kappa_{n}$ is bounded away from 0 and $2 \pi$ ), bounded 
by $C j^{-6}$ (from the fact that $a_{n+1}^{2}-a_{n}^{2} \sim j^{-6}$ ). Since there are $2 j^{4}$ subblocks in $\widetilde{B}_{j} \cup \widetilde{D}_{j}$, we see that

$$
\left|\sum_{n=1}^{N} Y_{n}\right| \leq \sum_{j}\left(2 j^{4}\right)\left(C j^{-6}\right)<\infty
$$

\section{The NTZ Argument}

Here we begin with the key lemma of Nevai-Totik-Zhang [34 and apply it to extend the result of Zhang [50] to allow approach to an isospectral torus.

Proposition 6.1 ([34]). For any positive $r$, any $\theta, \varphi \in[0,2 \pi]$, and $L$,

$$
\frac{12}{L} \sum_{j=0}^{L-1}\left|1-r e^{i(j \theta+\varphi)}\right|^{2} \geq\left|1-r e^{i \varphi}\right|^{2}
$$

Remarks. 1. [34] allow general $p>0$ where we take $p=2$; but for $p=2$, their constant is 32 , not 12 .

2. We include a proof at the end of this section for the reader's convenience and because we want to emphasize the concepts in the context of what we cannot do in the next section.

3. For $\theta$ not near 0 or $2 \pi$, the idea behind a bound of this form is the same as the idea behind Lemma 5.4. As $\theta \rightarrow 0$ or $2 \pi$, for this argument to work, the constant 12 has to be replaced by larger and larger numbers. The idea for small $\theta$ is instead to use the fact that enough terms need to be close to the initial one.

Corollary 6.2 ([50]). Let $A$ be a $2 \times 2$ matrix with

$$
\operatorname{det}(A)=1 \quad|\operatorname{Tr}(A)| \leq 2
$$

Then for any vector $v \in \mathbb{C}^{2}$ (with $v=\left(v_{1}, v_{2}\right)$ the components of $\left.v\right)$,

$$
\left|\left(A^{L-1} v\right)_{1}\right|^{2} \leq \frac{12}{L} \sum_{j=0}^{L-1}\left|\left(A^{j} v\right)_{1}\right|^{2}
$$

Proof. If $A$ obeys (6.2), so does $B=A^{-1}$, and if $w=A^{L-1} v$, (6.3) is equivalent to

$$
\left|w_{1}\right|^{2} \leq \frac{12}{L} \sum_{j=0}^{L-1}\left|\left(B^{j} w\right)_{1}\right|^{2}
$$

so we need only prove (6.4). 
Any $B$ obeying (6.2) is a limit of $B$ 's with $|\operatorname{Tr}(B)|<2$, so we can suppose

$$
\operatorname{det}(B)=1 \quad|\operatorname{Tr}(B)|<2
$$

In that case, $B$ is diagonalizable and has eigenvalue $e^{ \pm i \theta}$ with $2 \cos (\theta)=$ $\operatorname{Tr}(B)$.

In particular, for any $v$,

$$
\left(B^{\ell} v\right)_{1}=\alpha e^{i \ell \theta}+\beta e^{-i \ell \theta}
$$

for some $\alpha, \beta$. By replacing $\theta$ by $-\theta$, we can suppose $\alpha \neq 0$ (if $\alpha=$ $\beta=0$, (6.4) is trivial!). Write $-\beta / \alpha=r e^{-i \varphi}$. Then (6.4) is equivalent to (after multiplying by $|\alpha|^{-2}$ )

$$
\left|1-r e^{-i \varphi}\right|^{2} \leq \frac{12}{L} \sum_{j=0}^{L-1}\left|1-r e^{-i(2 j \theta+\varphi)}\right|^{2}
$$

which, after a change of names of $\theta, \varphi$, is (6.1).

Recall that if $\left\{a_{n}, b_{n}\right\}_{n=1}^{\infty}$ are Jacobi parameters, a two-sided set $\left\{a_{n}^{(r)}, b_{n}^{(r)}\right\}_{n=-\infty}^{\infty}$ is called a right limit if for some $m_{j} \rightarrow \infty$ and all $n=0, \pm 1, \ldots$,

$$
a_{m_{j}+n} \rightarrow a_{n}^{(r)} \quad b_{m_{j}+n} \rightarrow b_{n}^{(r)}
$$

If $\sup _{n}\left(\left|a_{n}\right|+\left|b_{n}\right|\right)<\infty$, there are right limits by compactness and, indeed, any sequence $m_{k}$ has a subsequence defining a right limit. Right limits are described in [43, Ch. 7] and references quoted there.

Any finite gap set

$$
\mathfrak{e}=\left[\alpha_{1}, \beta_{1}\right] \cup \cdots \cup\left[\alpha_{\ell+1}, \beta_{\ell+1}\right]
$$

with

$$
\alpha_{1}<\beta_{1}<\alpha_{2}<\beta_{2}<\cdots<\beta_{\ell+1}
$$

defines an $\ell$-dimensional isospectral torus, $\mathcal{T}_{\mathfrak{e}}$, of almost periodic twosided Jacobi matrices, $J$ with $\sigma(J)=\mathfrak{e}$. $\mathcal{T}_{\mathfrak{e}}$ can be defined using minimal Herglotz functions ([43, Ch. 5]) or reflectionless requirements ([43, Ch. 7]). If $\rho_{\mathfrak{e}}$ is the potential theoretic equilibrium measure for $\mathfrak{e}$ (see, e.g., [44, 39]), we say $\mathfrak{e}$ is "periodic" if and only if each $\rho_{\mathfrak{e}}\left(\left[\alpha_{j}, \beta_{j}\right]\right)$ is rational; equivalently, all $J \in \mathcal{T}_{\mathfrak{e}}$ have a common period $p$.

The Nevai class for $\mathfrak{e}$ is defined to be those one-sided $J$ 's whose right limits are all in $\mathcal{T}_{\mathfrak{e}}$. For $\mathfrak{e}=[-2,2], \mathcal{T}_{\mathfrak{e}}$ has a single point (with period 1 !) and the Nevai class for $\mathfrak{e}$ is the usual Nevai class.

Theorem 6.3. If $J$ lies in the Nevai class for a periodic $\mathfrak{e}$, then the Nevai condition holds uniformly for $J$ on $\mathfrak{e}$.

Remark. If $J$ has a single $p$ element orbit, $J^{(r)} \in \mathcal{T}_{\mathfrak{e}}$, as right limits (i.e., $J$ is asymptotically periodic), this is a result of [50, 47]. 
Proof. Let $p$ be the period of $\mathfrak{e}$. We will prove that any $x_{n} \in \mathfrak{e}$ and any $L$

$$
\limsup _{n \rightarrow \infty} \frac{\left|p_{n}\left(x_{n}\right)\right|^{2}}{\sum_{j=n-p L}^{n}\left|p_{j}\left(x_{n}\right)\right|^{2}} \leq \frac{12}{L}
$$

from which

$$
\limsup _{n \rightarrow \infty} \frac{\left|p_{n}\left(x_{n}\right)\right|^{2}}{\sum_{j=0}^{n}\left|p_{j}\left(x_{n}\right)\right|^{2}}=0
$$

proving the claimed uniform Nevai condition.

Without loss, we can pass to a subsequence so that $x_{n} \rightarrow x_{\infty}$, so that the ratio in (6.11) still converges to the limsup, so that $a_{n+k} \rightarrow a_{k}^{(r)}, b_{n+k} \rightarrow b_{k}^{(r)}$ for some periodic right limit and so that $\left(p_{n}\left(x_{n}\right), p_{n}\left(x_{n-1}\right)\right) /\left\|\left(p_{n}\left(x_{n}\right), p_{n-1}\left(x_{n-1}\right)\right)\right\|$ has a limit in $\mathbb{C}^{2}$.

The transfer matrix over $p$ units starting at 0 for that $x_{\infty}$ is a matrix $A$ obeying (6.2). So, by (6.3),

$$
\limsup _{n \rightarrow \infty} \frac{\left|p_{n}\left(x_{n}\right)\right|^{2}}{\sum_{j=0}^{L-1}\left|p_{n-j p}\left(x_{n}\right)\right|^{2}} \leq \frac{12}{L}
$$

which implies (6.11).

We turn to the proof of Proposition 6.1. Without loss, we can (by taking complex conjugates) suppose

$$
0<\theta \leq \pi
$$

(since $\theta=0$ is trivial). There are three cases to consider:

Case 1. $L \leq 12$, which is trivial.

Case 2.

$$
\theta L \geq 2 \pi \quad L \geq 13
$$

Case 3.

$$
\theta L<2 \pi
$$

Proof of Proposition 6.1. Consider Case 2 first, expanding

$$
\sum_{j=0}^{L-1}\left|1-r e^{i(j \theta+\varphi)}\right|^{2}=L\left(1+r^{2}\right)-2 r \operatorname{Re}[X]
$$

where

$$
X=\frac{e^{i(L \theta+\varphi)}-e^{i \varphi}}{e^{i \theta}-1}
$$

SO

$$
|X| \leq \frac{1}{\frac{1}{2}\left|1-e^{i \theta}\right|}=\frac{1}{\left|\sin \left(\frac{\theta}{2}\right)\right|} \leq \frac{\pi}{\theta}
$$


since

$$
\inf _{0 \leq y \leq \pi}\left[\frac{\sin \left(\frac{y}{2}\right)}{y}\right]=\frac{1}{\pi}
$$

By (6.15), $\pi / \theta \leq L / 2$, so by (6.17),

$$
\begin{aligned}
\text { LHS of (6.1) } & \geq L\left(1+r^{2}\right)-L r \\
& \geq \frac{L}{2}\left(1+r^{2}\right) \\
& >6\left(1+r^{2}\right)
\end{aligned}
$$

(since $L>12$ ). Clearly,

$$
\text { RHS of }(6.1) \leq|1+r|^{2} \leq 2\left(1+r^{2}\right)
$$

so (6.1) holds in Case 2.

That leaves Case 3. We will consider $\varphi<0(\varphi>0$ is even easier $)$. Consider the $L$ points

$$
T=\{\varphi+j \theta\}_{j=0}^{L-1}
$$

Since $L \theta<2 \pi$, they do not make it back around the circle. Consider the three sets: $S_{1}=\left\{\eta \mid \varphi \leq \eta<\frac{\varphi}{2}\right\}, S_{2}=\left\{\frac{\varphi}{2} \leq \eta<0\right\}$, and $S_{3}=\left\{0 \leq \eta<-\frac{\varphi}{2}\right\}$. Clearly, \#( $\left.S_{1} \cap T\right) \geq \max \left(\#\left(S_{2} \cap T\right), \#\left(S_{3} \cap T\right)\right)$, so at most two-thirds of the points in $T$ lie in $S_{2} \cup S_{3}$.

By the lemma below, if $\eta \in T \backslash\left(S_{2} \cup S_{3}\right)$,

$$
\left|1-r e^{i \eta}\right|^{2} \geq \frac{1}{4}\left|1-r e^{i \varphi}\right|^{2}
$$

SO

$$
\text { LHS of }\left(\underline{6.1)} \geq \frac{L}{3}\left(\frac{12}{L}\right) \frac{1}{4}\left|1-r e^{i \varphi}\right|^{2}=\right.\text { RHS of (6.1) }
$$

\section{Lemma 6.4.}

$$
\inf _{\substack{\pi \geq|\eta| \geq\left|\frac{\varphi}{2}\right| \\ 0<r}} \frac{\left|1-r e^{i \eta}\right|}{\left|1-r e^{i \varphi}\right|} \geq \frac{1}{2}
$$

Proof. $\left|1-r e^{i \eta}\right| /\left|1-r e^{i \varphi}\right|$ is invariant under $r \rightarrow r^{-1}$, so we can suppose $0<r \leq 1$. Moreover, $\left|1-r e^{i \eta}\right|$ is invariant under $\eta \rightarrow-\eta$ and increasing in $\eta$ for $0<\eta<\pi$, so the inf occurs at $\eta=\frac{\varphi}{2}$.

A straightforward calculation shows $\left|1-r e^{i \varphi / 2}\right| /\left|1-r e^{i \varphi}\right|$ is decreasing in $r$ in $r \in(0,1]$, so the inf is $\left|1-e^{i \varphi / 2}\right| /\left|1-e^{i \varphi}\right|=\left|\sin \left(\frac{\varphi}{4}\right)\right| /\left|\sin \left(\frac{\varphi}{2}\right)\right|=$ $1 /\left|2 \cos \left(\frac{\varphi}{4}\right)\right| \geq \frac{1}{2}$. 


\section{The Nevai Class of a General Finite Gap Set}

In this section, we will discuss the extension of Theorem 6.3 to general finite gap sets. We will only be able to prove the weaker result that the Nevai condition holds uniformly on compact subsets of $\mathfrak{e}^{\text {int }}$. In the next section, using different methods, we will prove the result uniformly on all of $\mathfrak{e}$.

We begin by noting the following abstraction of the argument we used in the proof of Theorem 6.3.

Proposition 7.1. Let $J$ be a half-line Jacobi matrix and let $\mathcal{R}$ be the set of its right limits. Let $K \subset \mathbb{R}$ be a compact set. For $v \in \mathbb{C}^{2}$ and $J^{(r)} \in \mathcal{R}$, let $u_{n}\left(v, J^{(r)}, z\right)$ solve

$$
a_{n}^{(r)} u_{n+1}+b_{n}^{(r)} u_{n}+a_{n-1}^{(r)} u_{n-1}=z u_{n}
$$

with

$$
\left(u_{0}, u_{1}\right)=\left(v_{1}, v_{2}\right)
$$

Suppose that for all $\varepsilon$, there is $N$ so that for all unit vectors $v \in \mathbb{C}^{2}$, all $J^{(r)} \in \mathcal{R}$, all $x_{0} \in K$, and all $n>N$,

$$
\frac{\left|u_{n}\left(v, J^{(r)}, x_{0}\right)\right|^{2}}{\sum_{j=0}^{n}\left|u_{j}\left(v, J^{(r)}, x_{0}\right)\right|^{2}} \leq \varepsilon
$$

Then $J$ obeys the Nevai condition uniformly on $K$.

Proposition 7.2. Let $J$ be a half-line Jacobi matrix obeying (1.14) and let $\mathcal{R}$ be the set of its right limits. Suppose that there is a compact subset $K \subset \mathbb{R}$ such that for each $x_{0} \in K$ and $J^{(r)} \in \mathcal{R}$, there is a solution $u_{n}^{+}\left(J^{(r)}, x_{0}\right)$ of (7.1) (with $\left.z=x_{0}\right)$ so that

$$
\begin{aligned}
& \sup _{n, x_{0}, J^{(r)}}\left|u_{n}^{+}\left(J^{(r)}, x_{0}\right)\right|<\infty \\
& \inf _{x_{0}, J^{(r)}} a_{0}^{(r)}\left|u_{1}^{+} \overline{u_{0}^{+}}-\overline{u_{1}^{+}} u_{0}^{+}\right|>0
\end{aligned}
$$

Then the Nevai condition holds for $J$ uniformly on $K$.

Remark. These are very strong conditions, but they hold in the finite gap case.

Proof. Define

$$
U_{n}\left(J^{(r)}, x_{0}\right)=\frac{1}{d\left(J^{(r)}, x_{0}\right)}\left(\begin{array}{cc}
u_{n+1}^{+} & \overline{u_{n+1}^{+}} \\
a_{n}^{(r)} u_{n}^{+} & a_{n}^{(r)} \overline{u_{n}^{+}}
\end{array}\right)
$$

where $d\left(J^{(r)}, x_{0}\right)$ is a square root of

$$
a_{n}^{(r)}\left(u_{n+1}^{+} \overline{u_{n}^{+}}-\overline{u_{n+1}^{+}} u_{n}^{+}\right)
$$


which is $n$-independent. Then $U_{n}$ is uniformly bounded in $x_{0} \in K$, $J^{(r)} \in \mathcal{R}, n$ by (17.4) /(7.5) and has determinant 1 , so the same is true of $U_{n}^{-1}$.

Moreover, the transfer matrix for $J^{(r)}$ is

$$
T_{n}=U_{n} U_{0}^{-1}
$$

so it is bounded in $n, J^{(r)}, x$, and has a bounded inverse. This shows

$$
\left|u_{n}\left(v, J^{(r)}, x_{0}\right)\right|^{2}+\left|u_{n+1}\left(v, J^{(r)}, x_{0}\right)\right|^{2}
$$

is uniformly bounded above and below as $v$ runs through unit vectors.

The ratio in (7.3) is thus uniformly bounded by $c / n$, so Proposition 7.1 is applicable.

Theorem 7.3. If $J$ lies in the Nevai class for a finite gap set $\mathfrak{e}$, then the Nevai condition holds uniformly on compact subsets of $\mathfrak{e}^{\text {int }}$.

Proof. In [6] (see also [43, Ch. 9]), Jost solutions are constructed on the isospectral torus, $\mathcal{T}_{\mathfrak{e}}$, that obey $(\underline{7.4}) /(\underline{7.5})$.

\section{Absence of Pure Points in Right Limits}

In this section, we want to note and apply the following:

Theorem 8.1. Let $J$ be a bounded half-line Jacobi matrix with (1.14) and let $\mathcal{R}$ be the set of its right limits. Let $\Xi$ be the set of $x_{0} \in \mathbb{R}$ so that for every $J^{(r)} \in \mathcal{R}$ and every nonzero solution $u_{n}$ of (7.1) with $z=x_{0}$, we have

$$
\sum_{n=-\infty}^{0}\left|u_{n}\right|^{2}=\infty
$$

Then

(i) The Nevai condition holds uniformly on any compact subset of $\Xi$.

(ii) If $\Xi$ contains $\sigma_{\mathrm{ess}}(J)$, then the Nevai condition holds uniformly on $\sigma(J)$.

We will provide a proof below. We first discuss some consequences.

Theorem 8.2. If $J$ lies in the Nevai class for a finite gap set $\mathfrak{e}$, then the Nevai condition holds uniformly on $\sigma(J)$.

Proof. In [6] (see also [43, Ch. 9]), it is proven that for any $J^{(r)}$ in the isospectral torus, $\mathcal{T}_{\mathfrak{e}}$, and any $x_{0} \in \mathfrak{e}^{\text {int }}$, every solution is almost periodic; and for $x_{0} \in\left\{\alpha_{j}, \beta_{j}\right\}_{j=1}^{\ell+1}$, every solution is the sum of an almost periodic function and $n$ times an almost periodic function. Nonzero almost periodic functions obey (8.1) and $\sigma_{\text {ess }}(J)=\mathfrak{e}$, so Theorem 8.1 is applicable. 
There is a class of whole-line stochastic Jacobi matrices called subshifts, with work reviewed in [8]. The most famous is the Fibonacci model which has $\left(\chi_{I}=\right.$ characteristic function of the set $\left.I\right)$

$$
a_{n} \equiv 1 \quad b_{n} \equiv \chi_{[1-\alpha, 1]}\left((n \alpha+\theta)_{\bmod 1}\right)
$$

where $\alpha=\frac{1}{2}(\sqrt{5}-1)$ and $\theta$ is a parameter (e.g., 0$)$. The name comes from the fact that the transfer matrix, $T_{n}$, has special properties when $n$ is a Fibonacci number. Damanik-Lenz [10] showed that there are no solutions $\ell^{2}$ at $-\infty$ for any $\theta$ and any $x_{0}$ in the spectrum, and it is not hard to see the right limits for any half-line Fibonacci problem are again Fibonacci models or such models modified at a single site. Thus, Theorem 8.1 is applicable, and

Theorem 8.3. Any Fibonacci model restricted to a half line obeys the Nevai condition uniformly on the spectrum.

Remark. Results of Damanik-Killip-Lenz [9] allow extension of this to general Sturmian models.

Theorem 8.1] was motivated by our trying to understand Szwarc [47]. He noted that one could use results of Nevai [32] on weak limits of the measure

$$
\sum_{j} \frac{p_{n}\left(x_{j}^{(n+1)}\right)^{2}}{\sum_{k=0}^{n} p_{k}\left(x_{j}^{(n+1)}\right)^{2}} \delta_{x_{j}^{(n+1)}}
$$

where $x_{j}^{(n+1)}$ are the solutions of

$$
p_{n+1}\left(x_{j}^{(n+1)}\right)=0
$$

that is, the ratios in (1.11) are weights in some natural measures. Thus, a failure of (1.11) should imply a suitable half-line limit has a pure point and that is what is forbidden by (8.1). We begin with:

Lemma 8.4. Let $J_{n ; F}$ be the $n \times n$ truncated Jacobi matrix. Then the spectral measure of $J_{n+1 ; F}$ and vector $\delta_{n+1}$ is (8.2) where the $x_{j}^{(n+1)}$ solve (8.3).

Proof. It is well known (see [43]) that

$$
\operatorname{det}\left(x-J_{n+1 ; F}\right)=P_{n+1}(x)
$$

so the eigenvalues are the solutions of (8.3). The unnormalized eigenvector for $x_{j}^{(n+1)}$ is $v_{k}$, given by

$$
v_{k}=p_{k-1}\left(x_{j}^{(n+1)}\right)
$$

so (8.2) has the squares of the normalized components for $\delta_{n+1}$. 
Lemma 8.5. Let $J_{F}^{(n)}$ be a family of $m_{n} \times m_{n}$ finite Jacobi matrices with coefficients associated to $\left\{a_{j}^{(n)}\right\}_{j=1}^{m_{n}-1} \cup\left\{b_{j}^{(n)}\right\}_{j=1}^{m_{n}}$. Suppose

(i) $m_{n} \rightarrow \infty$

(ii)

$$
\sup _{j, n}\left|a_{j}^{(n)}\right|+\left|b_{j}^{(n)}\right|<\infty
$$

(iii) For each fixed $j$,

$$
a_{j}^{(n)} \rightarrow a_{j}^{(\infty)} \quad b_{j}^{(n)} \rightarrow b_{j}^{(\infty)}
$$

Let $J^{(\infty)}$ be the infinite Jacobi matrix with parameters $\left\{a_{j}^{(\infty)}, b_{j}^{(\infty)}\right\}_{j=1}^{\infty}$. Let $d \rho^{(n)}$ be the spectral measure for $J_{F}^{(n)}$ and $\delta_{1}$, and $d \rho^{(\infty)}$ for $J^{(\infty)}$ and $\delta_{1}$. Then

$$
\underset{n \rightarrow \infty}{\mathrm{W}-\lim _{n \rightarrow \infty}} d \rho^{(n)}=d \rho^{(\infty)}
$$

Remark. This generalizes Theorem 3 in [32, p. 17].

Proof. Extend $J_{F}^{(n)}$ to an infinite matrix by setting all other matrix elements to 0 . Then (8.6) implies that

$$
\sup _{n}\left\|J_{F}^{(n)}\right\|<\infty
$$

and (8.7) implies that for any finite support vector, $v$,

$$
\left\|\left(J_{F}^{(n)}-J^{(\infty)}\right) v\right\| \rightarrow 0
$$

It follows that

$$
\mathrm{s}-\lim J_{F}^{(n)}=J^{(\infty)}
$$

So, by (8.9),

$$
\operatorname{s-lim}\left(J_{F}^{(n)}\right)^{k}=\left(J^{(\infty)}\right)^{k}
$$

for all $k$. Thus,

$$
\lim \left\langle\delta_{1},\left(J_{F}^{(n)}\right)^{k} \delta_{1}\right\rangle=\left\langle\delta_{1},\left(J^{(\infty)}\right)^{k} \delta_{1}\right\rangle
$$

So

$$
\lim \int x^{k} d \rho^{(n)}=\int x^{k} d \rho^{(\infty)}
$$

from which (8.8) follows.

Proof of Theorem 8.1. Let $\widetilde{K}$ be a compact subset of $\Xi$. If (1.11) does not hold uniformly, we can find $n(j) \rightarrow \infty, x_{j} \in \widetilde{K}$, and $\varepsilon>0$ so that for all $j$,

$$
\frac{p_{n(j)}\left(x_{j}\right)^{2}}{\sum_{k=0}^{n(j)} p_{k}\left(x_{j}\right)^{2}} \geq \varepsilon
$$


By passing to a subsequence, we can suppose

$$
x_{j} \rightarrow x_{\infty} \in \widetilde{K}
$$

Notice that $(\underline{8.15})$ implies

$$
p_{n(j)-1}\left(x_{j}\right)^{2} \leq \sum_{k=0}^{n(j)} p_{k}\left(x_{j}\right)^{2} \leq \varepsilon^{-1} p_{n(j)}\left(x_{j}\right)^{2}
$$

Define $\tilde{b}_{n(j)+1}$ by

$$
a_{n(j)} p_{n(j)-1}\left(x_{j}\right)+\left(\tilde{b}_{n(j)+1}-x_{j}\right) p_{n(j)}\left(x_{j}\right)=0
$$

Thus, for the OPs with Jacobi parameters $\left(a_{1}, \ldots, a_{n(j)}\right)$, $\left(b_{1}, \ldots, b_{n(j)}, \tilde{b}_{n(j)+1}\right)$, we have

$$
\tilde{p}_{n(j)+1}\left(x_{j}\right)=0
$$

Moreover, by (8.18) and (8.17),

$$
\lim \sup \left|\tilde{b}_{n(j)+1}\right| \leq \varepsilon^{-1 / 2} \sup _{k}\left|a_{k}\right|+\sup _{k}\left|x_{k}\right|
$$

is finite.

Let $J_{F}^{(j)}$ be the Jacobi matrix which is $(n(j)+1) \times(n(j)+1)$ with $a_{n(j)}, a_{n(j)-1}, \ldots, a_{1}$ off diagonal and $\tilde{b}_{n(j)+1}-x_{j}+x_{\infty}, b_{n(j)}-x_{j}+$ $x_{\infty}, \ldots, b_{1}-x_{j}+x_{\infty}$ on diagonal. By (8.15) and Lemma 8.4 (turning $J_{n(j)+1 ; F}$ on its head!), the spectral measure for $J_{F}^{(j)}, \delta_{1}$ has a pure point at $x_{\infty}$ of mass at least $\varepsilon$.

By passing to a further subsequence, we can suppose for all $q$ that $a_{n(j)+q} \rightarrow a_{q}^{(r)}, b_{n(j)+q} \rightarrow b_{q}^{(r)}$ for some right limit, $J^{(r)}$, and, by (8.20) and a further subsequence, that $\tilde{b}_{n(j)+1} \rightarrow \tilde{b}_{1}^{(r)}$.

The coefficients of $J_{F}^{(j)}$ clearly obey (8.6) and there is a $J^{(\infty)}$ so (8.7) holds. This is given by the reversed left side of $J^{(r)}$ (from $-\infty$ to 1 ), with $b_{1}^{(r)}$ replaced by $\tilde{b}_{1}^{(r)}$.

For any positive function $f$,

$$
\int f(y) d \rho^{(n)}(y) \geq \varepsilon f\left(x_{\infty}\right)
$$

So, by (8.8),

$$
\int f(y) d \rho^{(\infty)}(y) \geq \varepsilon f\left(x_{\infty}\right)
$$

Thus,

$$
\rho^{(\infty)}\left(\left\{x_{\infty}\right\}\right) \geq \varepsilon
$$


and $x_{\infty}$ is an eigenvalue of $J^{(\infty)}$. Thus, $J^{(r)}$ has an eigensolution at $x_{\infty}$ which is $\ell^{2}$ at $-\infty$, violating (8.1). This proves statement (i) of Theorem 8.2 .

For (ii), by passing to a subsequence, (8.16) holds for some $x_{\infty}$. If $x_{\infty}$ is an isolated eigenvalue, $x_{j}$ must be equal to $x_{\infty}$ for large $j$, so $\limsup \left|p_{j}\left(x_{\infty}\right)\right|>0$, violating the condition that $x_{\infty}$ is an isolated eigenvalue. If $x_{\infty} \in \sigma_{\text {ess }}(J)$, the argument in the first part produces a contradiction. Thus, (ii) is proven.

\section{Some Comments}

We end this paper with some final results and comments. The following must be well known in the ergodic theory community:

Proposition 9.1. Let $a_{n}$ be a sequence of reals and

$$
C_{n}=\frac{1}{n} \sum_{j=1}^{n} a_{j}
$$

If

exists, then

$$
\lim _{n \rightarrow \infty} C_{n}=C_{\infty} \neq 0
$$

$$
\frac{a_{n}}{n C_{n}} \rightarrow 0
$$

Proof. Since

$$
\begin{aligned}
a_{n} & =n C_{n}-(n-1) C_{n-1} \\
n^{-1} a_{n} & =C_{n}-C_{n-1}+n^{-1} C_{n-1}
\end{aligned}
$$

SO

$$
\frac{a_{n}}{n C_{n}}=\frac{C_{n}-C_{n-1}+n^{-1} C_{n-1}}{C_{n}}
$$

goes to zero if (9.2) holds.

It is an idea associated especially with Freud and Nevai (see [33]) that on the a.c. spectrum,

$$
\frac{1}{n} \sum_{j=0}^{n-1} p_{j}\left(x_{0}\right)^{2} \rightarrow \frac{\rho_{\infty}\left(x_{0}\right)}{w\left(x_{0}\right)}
$$

where $\rho_{\infty}$ is the density of zeros and $w$ is the a.c. part of the underlying measure, with concrete results both classical ([31, 48]) and recent ([1, 24, 25, 42, 49]).

Via Proposition 9.1, this gives several results on the Nevai condition. Totik's result [48] implies: 
Theorem 9.2. Let $d \rho$ have the form

$$
d \rho(x)=w(x) d x+d \rho_{\mathrm{s}}(x)
$$

with $d \rho_{\mathrm{s}}$ Lebesgue singular. Let $\mathfrak{e}$ be the essential support of $d \rho$ and suppose $\rho$ is regular for $\mathfrak{e}$. Suppose $I$ is an open interval in $\mathfrak{e}$ on which a Szegő condition holds:

$$
\int_{I} \log (w(x)) d x>-\infty
$$

Then for Lebesgue a.e. $x_{0} \in I$, the Nevai condition holds.

More recent work on uniform convergence [24, 42] implies

Theorem 9.3. Under the hypotheses of Theorem 9.2, if (9.9) is replaced by $w$ continuous on $I$ and $\inf _{I} w(x)>0$, then the Nevai condition holds on all of I uniformly on compact subsets of $I$.

Recent work on ergodic Jacobi matrices ([1]; see that paper for the definition of ergodic Jacobi matrices) implies

Theorem 9.4. Let $J_{\omega}$ be a family of ergodic Jacobi matrices obeying (1.14). Suppose $\Sigma_{\mathrm{ac}}$, the essential support of the a.c. spectrum, is nonempty. Then for a.e. $\omega, x_{0} \in \Sigma_{\mathrm{ac}}$, the Nevai condition holds.

We also want to make a comment regarding the unbounded case (where $\rho$ is not compactly supported). In this case, there exist measures, $\rho$, of various types (including pure point!) with $\lim _{n \rightarrow \infty} \frac{a_{n+1}^{2}\left[p_{n}\left(x_{0}\right)^{2}+p_{n+1}\left(x_{0}\right)^{2}\right]}{K_{n}\left(x_{0}, x_{0}\right)} \neq 0$ for $x$ in a set of positive $\rho$ measure. Indeed, the power law behavior of the generalized eigenfunctions in [3] and those associated with the absolutely continuous part of the measure in [11] imply the limit in these cases is actually $\infty$ for certain values of the relevant parameters. In the Introduction, it was noted that this means that truncations of the generalized eigenfunction do not form a sequence of approximate eigenvectors. Although (2.13) is still true in this case, Theorem 1.2 does not hold for measures that are not compactly supported, so this does not constitute a counterexample to Conjecture 1.4. In any case, it seems to be an interesting challenge to study the Nevai condition in the unbounded case.

Next, we turn to some remarks on Conjecture 1.4. It is standard to break $d \rho$ into three parts: a.c., pure point, and singular continuous. For compactly supported measures the Nevai condition always holds at pure points, since if $x_{0}$ is a pure point, $\sum_{j=0}^{\infty} p_{j}\left(x_{0}\right)^{2}<\infty$, so $p_{n}\left(x_{0}\right)^{2} \rightarrow$ 0 . For $d \rho_{\mathrm{c}}(=$ the continuous part of $d \rho)$ it is not hard to see that $\lim _{n \rightarrow \infty} \int \frac{p_{n}(x)^{2}}{\sum_{j=0}^{n} p_{j}(x)^{2}} d \rho_{\mathrm{c}}(x)=0$ so the issue is going from convergence of integrals to pointwise convergence. 
If $x_{0}$ is not a pure point but $\sup _{n}\left|p_{n}\left(x_{0}\right)\right|<\infty$, then since $\sum_{j=0}^{\infty} p_{j}\left(x_{0}\right)^{2}=\infty$, the Nevai condition holds. Thus, our Conjecture 1.4 is related to a famous conjecture of Steklov and what is sometimes called the Schrödinger conjecture [27]. There are known counterexamples to both conjectures (see [35] and [16]), but the counterexample in 35. has failure of boundedness at a single point and the counterexample in [16] does not seem to violate the Nevai condition, so Conjecture 1.4 could be true.

We note that the currently open version of the Schrödinger conjecture, that for a.e. $x_{0}$ in the essential support of the a.c. spectrum one has bounded eigenfunctions, would imply the Nevai condition a.e. on the essential support of the a.c. spectrum.

Finally, in connection with the example of Section 5, we would like to point out that we believe the following is true:

Conjecture 9.5. Let $q \in \mathbb{N}$, let $\left\{a_{n}, b_{n}\right\}_{n=1}^{\infty}$ be Jacobi parameters obeying

$$
\sum_{n=1}^{\infty}\left(a_{n+q}-a_{n}\right)^{2}+\left(b_{n+q}-b_{n}\right)^{2}<\infty
$$

and let $\mathcal{R}$ be the set of corresponding right limits. Then

$$
\Sigma_{\mathrm{ac}}=\bigcap_{\mathcal{R}} \sigma\left(J^{(r)}\right)
$$

where $\Sigma_{\mathrm{ac}}$ is the corresponding essential support of the a.c. spectrum.

We note that (9.10) implies $\mathcal{R}$ is made of $q$-periodic Jacobi matrices. The inclusion $\Sigma_{\text {ac }} \subset \bigcap_{\mathcal{R}} \sigma\left(J^{(r)}\right)$ follows from a general result of [21], so the point here is the inclusion in the other direction. Conjecture 9.5 generalizes a conjecture of Kaluzhny-Last [18], who make this conjecture for the special case where $\mathcal{R}$ consists of a single element. Denisov's result [13] establishes it for the special where $a_{n} \equiv 1$ and where the single element of $\mathcal{R}$ is the free Jacobi matrix (namely, $b_{n} \rightarrow 0$ ), proving an even earlier variant of this conjecture by Last [20]. Theorem [5.2 shows that Conjecture 9.5 holds for the Jacobi matrix of Example 5.1 (a special case where (9.10) holds for $q=1$ ), thus providing some level of confirmation for it.

\section{REFERENCES}

[1] A. Avila, Y. Last, and B. Simon, Bulk universality and clock spacing of zeros for ergodic Jacobi matrices with a.c. spectra, in preparation. 
[2] G. Boole, On the comparison of transcendents, with certain applications to the theory of definite integrals, Philos. Trans. Royal Soc. London 147 (1857), $745-803$.

[3] J. Breuer, Spectral and dynamical properties of certain random Jacobi matrices with growing parameters, to appear in Trans. Amer. Math. Soc.

[4] R. Carmona, One-dimensional Schrödinger operators with random or deterministic potentials: New spectral types, J. Funct. Anal. 51 (1983), 229-258.

[5] M. Christ, A. Kiselev, and Y. Last, Approximate eigenvectors and spectral theory, in "Differential Equations and Mathematical Physics," pp. 85-96, AMS/IP Stud. Adv. Math., 16, American Mathematical Society, Providence, RI, 2000.

[6] J. S. Christiansen, B. Simon, and M. Zinchenko, Finite gap Jacobi matrices, I. The isospectral torus, in preparation.

[7] H. L. Cycon, R. G. Froese, W. Kirsch, and B. Simon, Schrödinger Operators With Application to Quantum Mechanics and Global Geometry, Texts and Monographs in Physics, Springer, Berlin, 1987; corrected and extended reprint, 2008.

[8] D. Damanik, Strictly ergodic subshifts and associated operators, in "Spectral Theory and Mathematical Physics: A Festschrift in Honor of Barry Simon's 60th birthday," pp. 505-538, Proc. Sympos. Pure Math., 76.2, American Mathematical Society, Providence, RI, 2007.

[9] D. Damanik, R. Killip, and D. Lenz, Uniform spectral properties of onedimensional quasicrystals, III. alpha-continuity, Comm. Math. Phys. 212 (2000), 191-204.

[10] D. Damanik and D. Lenz, Uniform spectral properties of one-dimensional quasicrystals, I. Absence of eigenvalues, Comm. Math. Phys. 207 (1999), 687-696.

[11] D. Damanik and S. Naboko, Unbounded Jacobi matrices at critical coupling, J. Approx. Theory 145 (2007) 221-236.

[12] B. Davis, On the weak type $(1,1)$ inequality for conjugate functions, Proc. Amer. Math. Soc. 44 (1974), 307-311.

[13] S. Denisov, On a conjecture of Y. Last, preprint.

[14] J. Dombrowski, Quasitriangular matrices, Proc. Amer. Math. Soc. 69 (1978), 95-96.

[15] G. Freud, Orthogonal Polynomials, Pergamon Press, Oxford-New York, 1971.

[16] S. Jitomirskaya, Singular spectral properties of a one-dimensional Schrödinger operator with almost periodic potential, in "Dynamical Systems and Statistical Mechanics" (Moscow, 1991), pp. 215-254, Adv. Soviet Math., 3, American Mathematical Society, Providence, RI, 1991.

[17] S. Jitomirskaya and Y. Last, Power-law subordinacy and singular spectra, I. Half-line operators, Acta Math. 183 (1999), 171-189.

[18] U. Kaluzhny and Y. Last, Preservation of a.c. spectrum for random decaying perturbations of square-summable high-order variation, in preparation.

[19] A. Kiselev, Y. Last, and B. Simon, Modified Prüfer and EFGP transforms and the spectral analysis of one-dimensional Schrödinger operators, Comm. Math. Phys. 194 (1998), 1-45.

[20] Y. Last, Destruction of absolutely continuous spectrum by perturbation potentials of bounded variation, Commun. Math. Phys. 274 (2007), 243-252. 
[21] Y. Last and B. Simon, Eigenfunctions, transfer matrices, and absolutely continuous spectrum of one-dimensional Schrödinger operators, Invent. Math. 135 (1999), 329-367.

[22] Y. Last and B. Simon, The essential spectrum of Schrödinger, Jacobi, and CMV operators, J. Anal. Math. 98 (2006), 183-220.

[23] L. H. Loomis, A note on the Hilbert transform, Bull. Amer. Math. Soc. 52 (1946), 1082-1086.

[24] D. S. Lubinksy, A new approach to universality limits involving orthogonal polynomials, to appear in Ann. of Math.

[25] D. S. Lubinsky, Universality limits in the bulk for arbitrary measures on compact sets, to appear in J. Anal. Math.

[26] D. S. Lubinsky and P. Nevai, Sub-exponential growth of solutions of difference equations, J. London Math. Soc. (2) 46 (1992), 149-160.

[27] V. P. Maslov, S. A. Molchanov, and A. Ya. Gordon, Behavior of generalized eigenfunctions at infinity and the Schrödinger conjecture, Russian J. Math. Phys. 1 (1993), 71-104.

[28] A. Máté, P. Nevai, and V. Totik, What is beyond Szegő's theory of orthogonal polynomials?, in "Rational Approximation and Interpolation" (Tampa, FL, 1983), pp. 502-510, Lecture Notes in Math., 1105, Springer, Berlin, 1984.

[29] A. Máté, P. Nevai, and V. Totik, Extensions of Szegö's theory of orthogonal polynomials. II, Constr. Approx. 3 (1987), 51-72.

[30] A. Máté, P. Nevai, and V. Totik, Extensions of Szegö's theory of orthogonal polynomials. III, Constr. Approx. 3 (1987), 73-96.

[31] A. Máté, P. Nevai, and V. Totik, Szegö's extremum problem on the unit circle, Ann. of Math. 134 (1991), 433-453.

[32] P. Nevai, Orthogonal polynomials, Mem. Amer. Math. Soc. 18 (1979), no. 213, 185 pp.

[33] P. Nevai, Géza Freud, orthogonal polynomials and Christoffel functions. A case study, J. Approx. Theory 48 (1986), 167 pp.

[34] P. Nevai, V. Totik, and J. Zhang, Orthogonal polynomials: their growth relative to their sums, J. Approx. Theory 67 (1991), 215-234.

[35] E. A. Rakhmanov, Steklov's conjecture in the theory of orthogonal polynomials, Math. USSR-Sb. 36 (1980), 549-575; Russian original in: Mat. Sb. (N.S.) 108(150) (1979), 581-608, 640.

[36] C. Remling, The absolutely continuous spectrum of Jacobi matrices, preprint.

[37] B. Simon, Bounded eigenfunctions and absolutely continuous spectra for onedimensional Schrödinger operators, Proc. Amer. Math. Soc. 124 (1996), 3361-3369.

[38] B. Simon, Orthogonal Polynomials on the Unit Circle, Part 2: Spectral Theory, AMS Colloquium Series, 54.2, American Mathematical Society, Providence, RI, 2005.

[39] B. Simon, Equilibrium measures and capacities in spectral theory, Inverse Problems and Imaging 1 (2007), 713-772.

[40] B. Simon, Orthogonal polynomials with exponentially decaying recursion coefficients, in "Probability and Mathematical Physics," CRM Proc. and Lecture Notes 42 (2007), 453-463. 
[41] B. Simon, The Christoffel-Darboux kernel, to appear in "Perspectives in PDE, Harmonic Analysis and Applications" in honor of V. G. Maz'ya's 70th birthday, to be published in Proceedings of Symposia in Pure Mathematics.

[42] B. Simon, Two extensions of Lubinsky's universality theorem, to appear in J. Anal. Math.

[43] B. Simon, Szegö's Theorem and Its Descendants: Spectral Theory for $L^{2}$ Perturbations of Orthogonal Polynomials, in preparation; to be published by Princeton University Press.

[44] H. Stahl and V. Totik, General Orthogonal Polynomials, in "Encyclopedia of Mathematics and its Applications," 43, Cambridge University Press, Cambridge, 1992.

[45] G. Szegő, Orthogonal Polynomials, Amer. Math. Soc. Colloq. Publ., 23, American Mathematical Society, Providence, RI, 1939; third ed., 1967.

[46] R. Szwarc, A counterexample to subexponential growth of orthogonal polynomials, Constr. Approx. 11 (1995), 381-389.

[47] R. Szwarc, Uniform subexponential growth of orthogonal polynomials, J. Approx. Theory 81 (1995), 296-302.

[48] V. Totik, Asymptotics for Christoffel functions for general measures on the real line, J. Anal. Math. 81 (2000), 283-303.

[49] V. Totik, Universality and fine zero spacing on general sets, in preparation.

[50] J. Zhang, Relative growth of linear iterations and orthogonal polynomials on several intervals, Linear Algebra Appl. 186 (1993), 97-115. 